\title{
Structural Analysis of Respirasomes in Electron Transfer Pathway of Acidithiobacillus ferrooxidans: A Computer-Aided Molecular Designing Study
}

\author{
Mahesh Chandra Patra, Sukanta Kumar Pradhan, \\ Surya Narayan Rath, and Jitendra Maharana \\ BIF-Centre, Department of Bioinformatics, Orissa University of Agriculture and Technology, Bhubaneswar, Odisha 751003 , India \\ Correspondence should be addressed to Mahesh Chandra Patra; ml2mahesh@gmail.com
}

Received 18 June 2013; Accepted 17 July 2013

Academic Editors: O. Flomenbom, S. Taneva, and N. Weisleder

Copyright (C) 2013 Mahesh Chandra Patra et al. This is an open access article distributed under the Creative Commons Attribution License, which permits unrestricted use, distribution, and reproduction in any medium, provided the original work is properly cited.

\begin{abstract}
Acidithiobacillus ferrooxidans obtains its metabolic energy by reducing extracellular ferrous iron with either downhill or uphill electron transfer pathway. The downhill electron transfer pathway has been substantially explored in recent years to underpin the mechanism of iron respiration but, there exists a wide gap in our present understanding on how these proteins are organized as a supercomplex and what sort of atomic level interactions governs their stability in the iron respiratory chain. In the present study, we aimed at unraveling the structural basis of supermolecular association of respirasomes using protein threading, protein-protein docking, and molecular dynamics (MD) simulation protocols. Our results revealed that Phe312 of outer membrane cytochrome c plays a crucial role in diffusing electrons from heme $C$ group to Asp73 of rusticyanin. In line with the previous experimental results, His143 of rusticyanin was found to have a stable interaction with Glu121 of periplasmic cytochrome c4. Cytochrome c4 interacts with subunit B of cytochrome c oxidase through Lys146 and Thr148 of the conserved hydrophobic/aromatic motif 145-WKWTFSY-151 to attain stability during simulation. Phe468 of cytochrome c oxidase was found indispensable for stabilizing heme aa 3 during MD simulation. Taken together, we conclude that the molecular interactions of charged and hydrophobic amino acids present on the surface of each respirasome form a hypothetical electron wire in the iron respiratory supercomplex of $A$. ferrooxidans.
\end{abstract}

\section{Introduction}

Acidithiobacillus ferrooxidans is an extensively studied Gramnegative acidophilic chemolithoautotrophic bacterium adored for its remarkable ability to oxidize insoluble ferrous iron $\left(\mathrm{Fe}^{2+}\right)$ to soluble ferric iron $\left(\mathrm{Fe}^{3+}\right)$. This obligate prokaryote derives its metabolic energy by oxidizing reduced sulfur compounds or ores containing $\mathrm{Fe}^{2+}$ under acidic condition using intracellular $\mathrm{O}_{2}$ as oxidant [1]. The electrons released during oxidation of $\mathrm{Fe}^{2+}$ are transported across the cytoplasmic membrane either through a thermodynamically favorable "downhill" pathway for the reduction of $\mathrm{O}_{2}$ to $\mathrm{H}_{2} \mathrm{O}$ or pushed against the redox potential gradient along an "uphill" pathway to reduce $\mathrm{NAD}(\mathrm{P})^{+}$to $\mathrm{NAD}(\mathrm{P})(\mathrm{H})[2-4]$.

Unlike many other anaerobic respiratory chains, the bioenergetic metabolism of this organism involves several proteins with highest redox potential and is encoded by rus and petI operons for the downhill and uphill pathways, respectively $[4,5]$. The rus operon constitutes a set of eight genes encoding an outer membrane $(\mathrm{OM})$ cytochrome $\mathrm{c}$ (Cyc2), a periplasmic blue copper $(\mathrm{Cu})$ rusticyanin $(\mathrm{RcY})$, a periplasmic cytochrome $\mathrm{c} 4(\mathrm{Cycl})$, a putative $\mathrm{OM}$ protein of unknown function (ORF1), and an inner membrane (IM) aa3 Cox [5-8]. It has been demonstrated that expression of the rus operon at transcriptional and posttranscriptional level is specifically triggered by the presence of $\mathrm{Fe}^{2+}$ at the extracellular environment. Further, biochemical studies have established that $\mathrm{Fe}^{2+}$ is oxidized by high molecular Cyc2, present at the $\mathrm{OM}$, and electrons are transferred downward to $\mathrm{O}_{2}$ through periplasmic $\mathrm{RcY}$ and $\mathrm{Cycl}$, finally reaching inner membrane aa3 Cox [6, 9-12] for energy transduction.

Recently, it has been proposed that the proteins required for downhill electron transfer are organized as a supercomplex spanning OM, periplasm, and IM [13]. Amongst 
the four respirasomes of downhill pathway, crystal structures of periplasmic RcY $[14,15]$ and Cycl [16] have been determined, but experimental information on the structure of OM Cyc2 and IM Cox is yet to be elucidated. This limitation is hindering postulation of a valid model for the specific binding surfaces of respirasomes. However, a working model of binding interaction between $\mathrm{RcY}$ and $\mathrm{Cycl}$ has been proposed by Mukhopadhyay et al., who showed that water molecules are implicated in transferring electrons from RcY to $\mathrm{Cycl}$ [17-19]. It is intriguing to note that, despite more than 40 years of research, the structural features of Cyc2 and its interactions with RcY are poorly understood. In addition, the interaction between Cox and Cycl is still elusive. In this scenario, theoretical models for the unsolved structures of respirasomes would be highly beneficial to establish a crude hypothesis on the probable electron channel in $A$. ferrooxidans.

In the present context, we employed rigid-body proteinprotein docking and explicit membrane water simulation protocols to construct abstract models of the respirasomes (Cyc2, RcY, Cycl, and Cox) for identifying the domains likely to persuade transferring of electrons. In order to achieve this, first we constructed the three-dimensional (3D) model of Cyc2 using threading algorithms followed by docking with the crystal structure of RcY. Second, the Xray structure of $\mathrm{Cycl}$ was docked with $\mathrm{RcY}$ that is bound to Cyc2. Third, the docked homology models of CoxA and CoxB subunits were tied up against $\mathrm{Cycl}$ of the Cyc2-RcYCycl complex. Subsequently, the whole respirasome complex was subjected to molecular dynamics (MD) simulation for obtaining completely relaxed molecular conformations. The results thus obtained provided us a convincing picture of popular electron-wire model postulated to occur within downhill electron transfer pathway $[6,16]$.

\section{Materials and Methods}

2.1. Protein Threading of Cyc2. The primary sequence of Cyc2 protein (accession number: O33823) was obtained from the UniProtKB database. Template search using BLAST and PSIBLAST web servers [20] against Protein Data Bank (PDB) revealed that Cyc2 sequence had extremely low (10\% to $14 \%$ ) identity with all the solved structures available in PDB. At this low sequence identity, structure prediction using standard homology modeling protocol is prone to numerous structural errors. Therefore, the 3D model of Cyc2 was built with commonly and successfully employed fold recognition or threading approaches [21]. The target sequence was subjected to several fold recognition servers such as PHYRE [22], FUGUE [23], SAMT02 [24], pGenTHREADER [25] and two metaservers such as Genesilico [26] and Pcons [27]. The best hit of each server was compared against the secondary structure of the target protein predicted by PSIPREDv3.0 [28]. Our analysis revealed that the second top scored template (PDB ID: 2VSE [29]) suggested by pGenTHREADER had comparable secondary structure as that of Cyc2. The targettemplate pair-wise sequence alignment was performed using Discovery Studio v3.1 software (DS 3.1; Accelrys Software
Inc.). The alignment was carefully edited to comply with the sequence alignment proposed by pGenTHREADER. The Nterminal residues of $\mathrm{Cyc} 2$ corresponding to the standard signal sequence were truncated before constructing the model. Ten models of Cyc2 protein were generated using DS 3.1, and the best model was selected according to minimum discrete optimized potential energy (DOPE) score. The resulting Cyc2 3D model was optimized and refined by energy minimization with steepest descent integrator using GROMOS9643al force field of GROMACS software [30]. Stereochemical evaluation of the model was done in Auto Dep Input Tool (ADIT) validation server (http://validate.rcsb.org/), SAVeS server (http://nihserver.mbi.ucla.edu/SAVES/), and Z-score was calculated by ProSA-web [31].

2.2. Docking of heme $C$ with Cyc2. In A. ferrooxidans, heme $C$ group is attached to the $\mathrm{CXXCH}$ signature motif present at the $\mathrm{N}$ terminus of $\mathrm{Cyc} 2$ protein [32]. The heme $C$ group was obtained from NCBI's PubChem database [33], using the PubChem ID: 444125. The docking of heme $C$ with Cyc2 was performed by PatchDock server [34] using a clustering RMSD value of $1.5 \AA$, which is recommended for clustering protein-small molecule docking solutions. Top ten docking solutions were downloaded from the server, and the single best solution was selected according to highest geometric shape complementarity score [35]. The interaction of heme $C$ with its binding residues and correctness of docking were ascertained by visual inspection using graphical interface of DS 3.1.

2.3. Docking of Cyc2 with RcY. The X-ray structure of RcY was obtained from PDB using PDB ID: 2CAK [14]. The surface amino acids of Cyc2 and RcY were analyzed for charged residues that might form potential interacting interfaces. It was reported that the $\mathrm{Cu}$ atom of $\mathrm{RcY}$ interacts with heme $A$ of Cycl to transfer the electron [16]. Hence, it is reasonable to postulate that opposite surface residues of the $\mathrm{Cu}$ binding site must interact with Cyc2. Therefore, residues near the heme $C$ group of $\mathrm{Cyc} 2$ and residues opposite to $\mathrm{Cu}$ binding site of $\mathrm{RcY}$ were chosen as probable binding interface. The structure of $\mathrm{RcY}$ was corrected for errors like nonstandard names, alternate conformations, missing $\mathrm{N}$ and $\mathrm{C}$ termini, and incomplete atom sets in amino acids. Missing hydrogen atoms were added using DS 3.1 before subjecting to docking analysis. The docking of $\mathrm{Cyc} 2$ with $\mathrm{RcY}$ was performed with PatchDock. In order to get relevant solutions, potential binding site residues of $\mathrm{Cyc} 2$ and $\mathrm{RcY}$ were included in a text file and subjected to PatchDock for docking search stage. Residues surrounding the surface region near heme $C$ were considered as receptor binding site, while residues opposite to $\mathrm{Cu}$ site of $\mathrm{RcY}$ were specified as ligand binding site. The final clustering of docked complexes was done by specifying a clustering RMSD value of $4 \AA$, which is recommended for protein-protein docking. PatchDock implements different sets of parameters optimized for different types of complexes. We specified the "default complex" type, which is recommended for general purpose protein-protein docking 
experiments. The best docked conformations were ranked based on geometric shape complementarity score.

2.4. Docking of Cyc1 with Cyc2-RcY Complex. The crystal structure of $\mathrm{Cycl}$ was downloaded from PDB using the PDB ID: $1 \mathrm{H} 1 \mathrm{O}$ [16]. The structure preparation of $\mathrm{Cycl}$ was similar to $\mathrm{RcY}$ as described in the previous section. All hydrogen atoms were added before performing docking experiment using PatchDock. In this docking experiment, the Cyc2RcY complex was taken as receptor, and the Cycl structure was considered ligand. Cyc2 and RcY were represented by chains $\mathrm{A}$ and $\mathrm{B}$, respectively. The electrons are transferred from the $\mathrm{Cu}$ center of $\mathrm{RcY}$ to the heme $A$ group of $\mathrm{Cycl}$ through the interaction of charged residues on RcY and Cycl [16]. Therefore, the oppositely charged surfaces of both the proteins were supplied as binding sites to PatchDock server. Other docking parameters are same as those used for docking of Cyc2 and RcY.

2.5. Modeling and Docking of CoxA and CoxB. The 3D model for CoxA and CoxB subunits was constructed using respective atomic coordinates of the crystal structure of Paracoccus denitrificans (PDB ID: 1AR1 [36]). Sequence alignment and model building were performed with DS 3.1 software. Accuracy of the built models was confirmed by ADIT, SAVeS, and ProSA-web servers. Final docking of CoxAB dimer with $\mathrm{Cycl}$ of $\mathrm{Cyc} 2-\mathrm{RcY}-\mathrm{Cycl}$ complex was carried out in PatchDock using same parameters as described for protein-protein docking above. The complete molecular association of the respirasomes (Cyc2-RcY-Cyc1-CoxAB) was then energy minimized and optimized by MD simulation in explicit membrane aqueous environment before continuing further interaction studies.

2.6. MD Simulation. The proteins responsible for electron transfer in A. ferrooxidans are arranged in the form of an electron wire spanning OM, periplasm, and IM or cytoplasmic membrane [13]. Therefore, structural refinement of the docked complex was performed by placing the proteins in a two-membrane compartment system in such a manner that Cyc2 was partially integrated in $\mathrm{OM}$, while CoxAB dimer was embedded in IM. For membrane construction, preequilibrated palmitoyl-oleoyl-phosphoethanolamine (POPE) bilayer was obtained from the D. Peter Tieleman website [37]. InflateGRO methodology [38] was implemented for the insertion of the docked respirasome complex within the membrane bilayer. MD simulation was carried out with a hybrid force field containing Gromos96-53a6 [39] and Berger-lipid [40] parameters. Water layers were added to the simulation system using a van der Walls radius of $0.375 \AA$ for carbon atoms of lipid acyl chains, thus avoiding water molecules within hydrophobic core of the bilayers. A physiological ionic strength $(0.15 \mathrm{M})$ of counter ions was added to attain electroneutrality. The resultant system was energy minimized in GROMACS 4.5 simulation package for 10,000 steps constraining backbone atoms of the proteins with steepest descent integrator until convergence point at maximum force of $1000 \mathrm{kJmol}^{-1} \mathrm{~nm}^{-1}$ was observed. This was followed by 5,000 steps of conjugate gradient minimization without backbone constraints to obtain strain-free molecular conformations. The energy minimized structures were then divided into separate index groups for calculating energy values and other dynamic properties for individual proteins after MD simulation. Position restraints dynamics was performed by constraining backbone atoms of the proteins first in NVT ensemble for 100 ps followed by NPT ensemble for 1 ns. Finally, production run of $5 \mathrm{~ns}$ was performed without backbone constraints. The simulations were performed at $300 \mathrm{~K}$ using a time step of 0.002 ps. A distance cutoff of $12 \AA$ with switching at $10 \AA$ was used for van der Waals interactions. The particle mesh Ewald (PME) method was applied to treat electrostatic interactions. The trajectory data was saved at every 2,500 steps (corresponding to $5 \mathrm{ps),} \mathrm{generating} 1,000$ structures over $5 \mathrm{~ns}$ of simulation for analysis. Trajectory data was analyzed using VMD 1.8.7 [41] and gnuplot 4.2 (http://www.gnuplot.vt.edu/) programs. Molecular graphics and visualization were prepared using DS 3.1 and VMD software.

\section{Results and Discussion}

3.1. Construction of Cyc2 3D Model. In the downhill iron respiratory chain of $A$. ferrooxidans, electrons are transferred along a series of membrane-located and periplasmic soluble proteins which are arranged in the form of a supercomplex through direct protein-protein interactions spanning both $\mathrm{OM}$ and IM [13]. Cyc2 protein is the first electron carrier in the $\mathrm{Fe}^{2+}$ respiratory pathway [6] and is one of the most diverse kinds of protein molecules having extremely low sequence identity with the homologous proteins of known structures. Our sequence analysis of Cyc2 in $\mathrm{PDB}$ using BlastP tool revealed that all the resultant hits have $\sim 14 \%$ sequence identities, which is far below the minimum identity of $30 \%$ required for homology modeling [42]. Therefore, the task of template selection and target-template alignment was carried out by employing fold recognition or threading method. Previously, Schoonman et al. have suggested that the threading methods are able to predict more accurate models than comparative modeling in cases of low sequence identity $(<30 \%)$ [43]. Thus, based on the recent studies of successful implementation of threading approach [44-49], we carried out template search against the fold databases using several threading algorithms including PHYRE, FUGUE, pGenTHREADER, SAMT02, Genesilico, and Pcons metaservers. Amongst all the results of threading algorithms, only the mosquitocidal holotoxin from Bacillus sphaericus (2VSE) proposed by pGenTHREADER had similar secondary structure as that of Cyc2 with a $P$ value of 0.0006 , SolvE score of -4.2 , and net score of 48.983 amid a high confidence prediction range. Pairwise sequence alignment of 2VSE and Cyc2 is shown in Figure 1(a). Accuracy of the constructed model was validated by stereochemical analysis (dihedral angles; Phi/Psi) using Ramachandran plot [50], which indicated that $86.9 \%$ of residues fall in the most favored region, $12.2 \%$ of residues in the additional allowed region, $0.9 \%$ of residues in the generously allowed region, and no residues in the disallowed region 


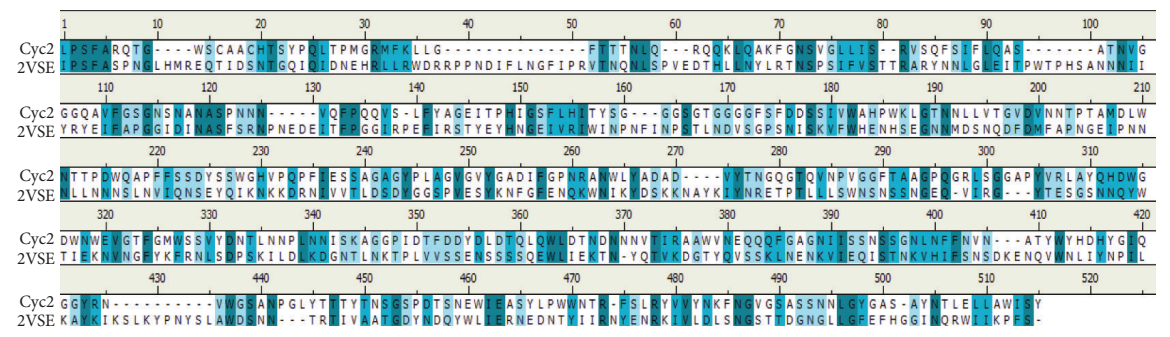

(a)

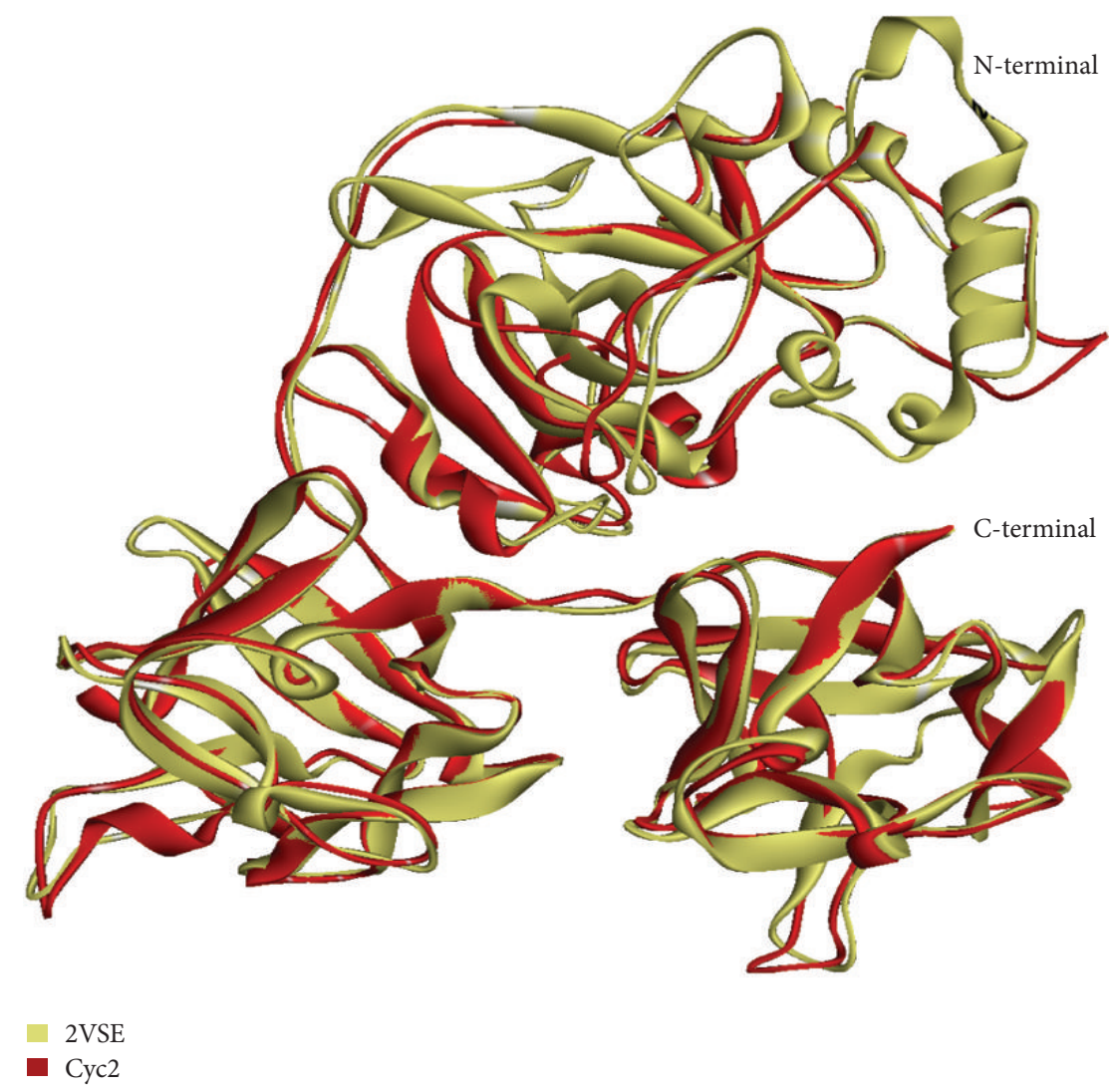

(b)

Figure 1: (a) Sequence alignment between Cyc2 (target) and 2VSE (template). Deep blue, light blue, and cyan colors refer to identical residues, conserved and semiconserved substitutions, respectively. (b) Schematic representation of modeled Cyc2 (yellow) superimposed to the template 2 VSE (red) with a backbone RMSD of $0.9 \AA$.

(Figure S1 of the Supplementary Material available online at http://dx.doi.org/10.1155/2013/295718). The resulting $Z$ score of Cyc2 was found to be -7.25 (Figure S2), which is comparable to that of the known X-ray structures of similar size available in PDB. For additional verification of the compatibility of the generated model with its primary sequence, Verify 3D was implemented, which revealed that $91.84 \%$ of the residues had an average 3D-1D score $>0.2$, indicating a good quality model. Secondary structure prediction of the modeled Cyc2 protein revealed predominant presence of beta sheets and random coils with few alphahelical segments (Figure S3). Further, the modeled protein was structurally analyzed by superimposing on the template using $\mathrm{C} \alpha$-atoms. Although the sequence identity between Cyc2 and 2VSE was only $14 \%$, the low RMSD value of $0.9 \AA$ indicated that the quality of the generated model is reasonably good having entire beta sheets and coil regions with similar spatial orientations (Figure 1(b)). With this well-validated geometry, the modeled Cyc2 structure was considered for further analysis. Hydropathy plot analysis suggests that Cyc2 contains no putative transmembrane (TM) segments (Figure S4). Hence, it is plausible that Cyc2 is partially integrated in the $\mathrm{OM}$ and the heme $\mathrm{C}$ containing portions; that is, $\mathrm{CXXCH}$ signature motif at the $\mathrm{N}$ terminus is likely to accept electrons released during the oxidation of $\mathrm{Fe}^{2+}$. The $\mathrm{OM}$ localization of Cyc2 finds support from some earlier study showing presence 
of cytochrome $\mathrm{c}$ in the $\mathrm{OM}$ region of some neutrophilic microorganisms deriving metabolic energy from insoluble metals and minerals [51-54].

3.2. Interaction between heme $C$ and $C y c 2$. The heme-containing proteins carry out numerous biological functions due to the different interactions between heme group and surrounding amino acids. In $A$. ferrooxidans, the heme $C$ group of Cyc2 performs one of the most important biological functions by binding and transporting electrons released during oxidation of $\mathrm{Fe}^{2+}$. Hence, we studied the interaction of heme $C$ with neighboring residues. In order to accomplish this, docking of heme $C$ was carried out in the binding site of Cyc2. The heme $C$ was docked close to the conserved heme binding motif, $43-\mathrm{CXXCH}-47$ that is present in all heme-containing proteins, and His residue of this motif is found covalently attached to the central iron atom of the heme group [32]. A close inspection of docked complex indicated that His 47 of $43-\mathrm{CXXCH}-47$ is present at a distance of $2.5 \AA$ from the central Fe atom of heme $C$ (Figure 2(a)), and in biological conditions a covalent bond is expected between the Fe and imidazole ring of His47 [55]. In addition, heme $C$ was observed to be surrounded by a large number of hydrophobic residues, namely, Phe227, Tyr441, Phe312, Tyr50, Trp436, Leu135, Phe136, and Trp445. The two carboxyl groups $\left(\mathrm{COO}^{-}\right)$of heme $C$ form strong hydrogen bonds $(\mathrm{H}-$ bonds) with the side chains of Glu438 and Asn358 (Figure S5). The stability of heme $C$ was further confirmed after observing a strong $\mathrm{H}$-bond network around its $10 \AA$ radius (Figure S5). We found a number of intermolecular $\mathrm{H}$-bonds between the residues of $\mathrm{Cyc} 2$ and heme $C$ that has been shown in Table S1. The heme $C$ contains two sulfur atoms, $\mathrm{S} 1$ and S2, of which S1 interacts with Thr48 through H-bonds (Figure 3). The heme $C$ is found entangled in a hydrophobic pocket at the surface of the protein. Because the hydrophobic region should always be excluded from solvent environment, we can speculate that the heme binding pocket could be the most probable binding surface for RcY, thus indicating a probable path of electron transfer to RcY (Figure 2(b)).

3.3. Molecular Interaction between $C y c 2$ and $R c Y$. RcY is the intermediate electron carrier in the downhill $\mathrm{Fe}^{2+}$ pathway of A. ferrooxidans. In the initial phase of energy transduction, Cyc2 spontaneously transfers electrons to RcY. Therefore, we examined the intermolecular interactions of $\mathrm{Cyc} 2$ and $\mathrm{RcY}$ to gain more insight about their interacting surfaces. First, we ascertained their conformational stability in bound condition during MD simulation. As can be seen from Figure 3, Cyc2 and RcY displayed backbone (C-N-CA) RMSDs of $0.44 \mathrm{~nm}$ and $0.12 \mathrm{~nm}$, respectively. The low RMSD value of RcY as compared to Cyc2 indicates that RcY is structurally more rigid than $\mathrm{Cyc} 2$, probably because it is bound on either side by $\mathrm{Cyc} 2$ and $\mathrm{Cycl}$ in the respiratory supercomplex. On the other hand, Cyc2 being freed at extracellular end or possibly due to its larger size (449 amino acids) took longer equilibration time, but it eventually attained stability for rest of the simulation.
Average coordinates Cyc2 and RcY complex were extracted from the most stable portion of their respective dynamics trajectories. A close analysis revealed that Cyc2 interacts with RcY mainly through hydrophobic and partly with electrostatic interactions. A single $\mathrm{H}$-bond (interatomic distance of $2.7 \AA$ ) was observed between main chain CO of Gly310 from Cyc2 and side chain $\mathrm{OH}$ of Thr126 from RcY (Figure 2(b)). By carefully observing the interacting surface of RcY, we can say that Asp73, the only negatively charged residue properly situated on the opposite surface of $\mathrm{Cu}$ center, could be the most probable component to facilitate electron transfer from heme $C$ group of $\mathrm{Cyc} 2$ to the $\mathrm{Cu}$ atom of $\mathrm{RcY}$ (Figures 2(b) and 4(a)). Abergel et al. [16] have previously reported that in RcY-Cycl complex, the negatively charged Glu121 of $\mathrm{Cycl}$ is crucial for the stability during the electron transfer. Similarly, in our Cyc2-RcY interaction model, the negatively charged Asp73 of RcY might be critical for the stability of the complex. Further, previous reports have indicated that Asp73 might serve as one of the $\mathrm{Cu}$ coordinating ligands [56]. In contrast, subsequent site-specific mutants and gene expression results revealed that His 85 acts as the $\mathrm{Cu}$ coordinating ligand [57]. In our present docking model, we observed that Asp73 is actually situated at the opposite surface of $\mathrm{Cu}$ site in $\mathrm{RcY}$ and thus is unlikely a coordinate $\mathrm{Cu}$ atom. From these observations, it can be deduced that Asp73 might be transferring electrons to the $\mathrm{Cu}$ center through a passage made by the core hydrophobic residues. Further, the presence of Phe 312 of Cyc2 at intermediate position between heme $C$ and Asp73 could be presumed an important factor influencing electron transfer from the donor site of heme $C$ to the acceptor site of Asp73 (Figure 2(b)). Previous studies on the cytochromes had clearly demonstrated the significance of phenylalanine/tyrosine residues in mediating electron transfer $[58,59]$. A close visual inspection of the core region of RcY structure after MD simulation allowed us to observe that several hydrophobic interactions comprising the residues Phe125, Val74, Phe76, Phe54, Phe87, and Trp7 form a channel required for electron transfer to the $\mathrm{Cu}$ center (Figure 4(a)). These observations suggest that hydrophobic interactions potentially play crucial roles in upholding the structure and function of $\mathrm{Cyc} 2-\mathrm{RcY}$ complex. The stable association between Cyc2 and RcY observed by proteinprotein docking is in congruence with recently reported far-Western blotting experiments showing direct interaction between the two proteins in the respiratory supercomplex [13].

3.4. Interaction between $R c Y$ and Cycl. Cycl is the third electron acceptor in the downhill electron pathway of $A$. ferrooxidans, which receives the electron from RcY. After determining the stable interaction between Cyc2 and RcY, we analyzed the binding interface between RcY and Cycl. To understand these interactions, first we investigated the interaction of amino acids around the $\mathrm{Cu}$ center of RcY during MD simulation. The crystal structure of RcY was found to have ten pairs of electronegative and electropositive atoms within a distance of $3.5 \AA$ around the $\mathrm{Cu}$ atom. We monitored breaking and formation of this $\mathrm{H}$-bond network throughout 


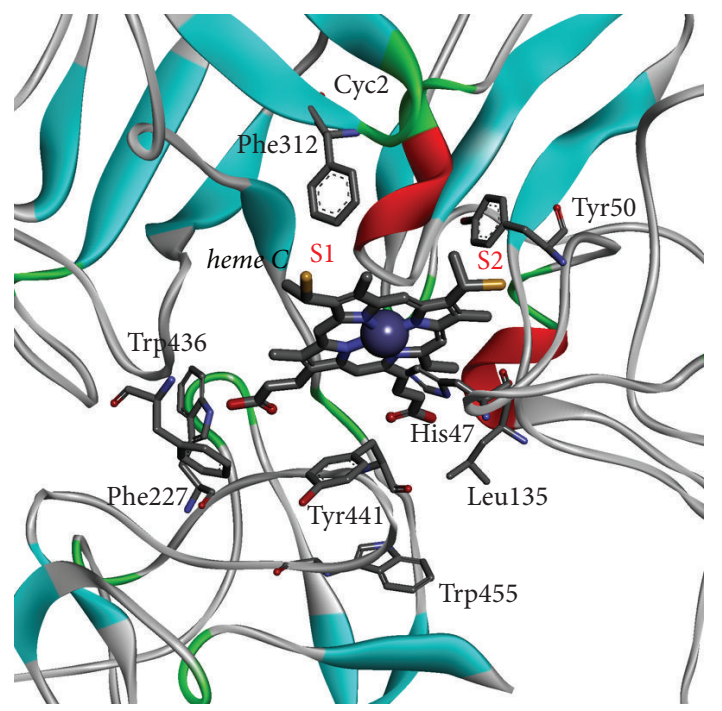

(a)

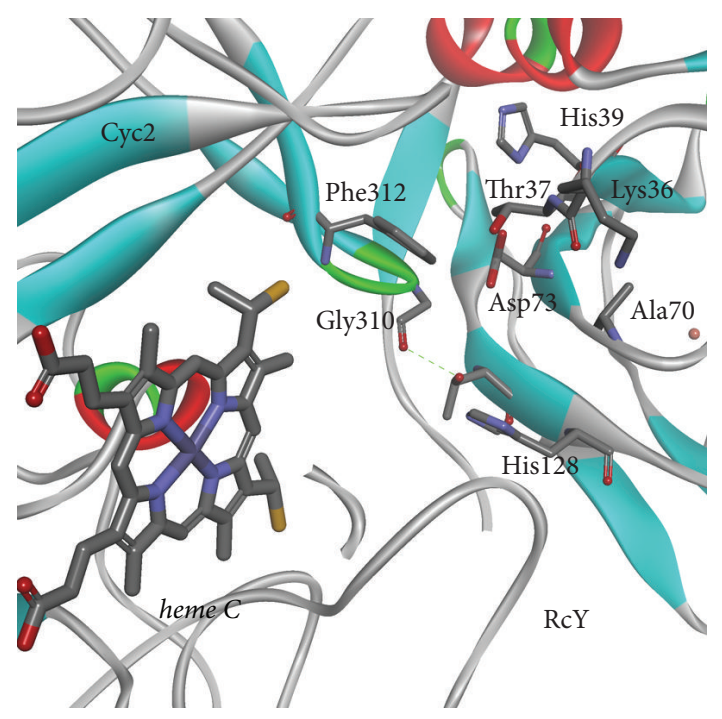

(b)

FIGURE 2: (a) Crucial amino acids of Cyc2 taking part in hydrophobic interactions with heme $C$. The active site hydrophobic residues stabilizing heme $C$ have been represented as stick models. (b) The graphical representation of favorable electron pathway from heme $C$ group of Cyc2 to Asp73 of RcY.

TABLE 1: Calculation of H-bonds between the residues around $3.5 \AA$ of $\mathrm{Cu}$ atom within RcY protein during MD simulation.

\begin{tabular}{|c|c|c|c|c|c|}
\hline RcY (residues) & Donor & Acceptor & RcY (residues) & Distance $(\AA)$ & Average duration (ns) \\
\hline Ser53 & OG & $\mathrm{O}$ & Pro52 & 2.6 & 3 \\
\hline Asn 80 & $\mathrm{~N}$ & $\mathrm{O}$ & Val45 & 2.7 & 2.9 \\
\hline Asn 80 & ND2 & NE2 & His85 & 2.9 & 3.9 \\
\hline His85 & $\mathrm{N}$ & OG & Ser112 & 2.7 & 4 \\
\hline Ser86 & $\mathrm{N}$ & SG & Cys138 & 3.1 & 4.9 \\
\hline Ser112 & $\mathrm{N}$ & $\mathrm{O}$ & Gly84 & 2.8 & 2.9 \\
\hline Ser112 & OG & $\mathrm{O}$ & His85 & 2.5 & 4.1 \\
\hline Ile40 & $\mathrm{N}$ & $\mathrm{O}$ & Cys138 & 3.0 & 1.6 \\
\hline His143 & $\mathrm{N}$ & $\mathrm{O}$ & Ile40 & 2.9 & 4.5 \\
\hline Met148 & $\mathrm{N}$ & $\mathrm{O}$ & Pro52 & 3.1 & 4.3 \\
\hline
\end{tabular}

the $5 \mathrm{~ns} \mathrm{MD}$ trajectories. As can be seen from Table 1, seven $\mathrm{H}$-bonds persisted for longer than $3 \mathrm{~ns}$, and interestingly, all $\mathrm{Cu}$ coordinating ligands except $\mathrm{Cycl} 38$ continued to form $\mathrm{H}$ bonds for longer than $4 \mathrm{~ns}$. Similar to the calculated RMSD of RcY, the crystal structure of $\mathrm{Cycl}$ showed a low RMSD profile $(0.19 \mathrm{~nm})$ during $\mathrm{MD}$ simulation (Figure 3$)$. Since Cycl is bound on one side by RcY and on the other side by Cox, it remained reasonably stable. Average coordinates were obtained from the stable dynamics trajectories, and $\mathrm{H}$ bonds were analyzed. The side chain amino group $\left(\mathrm{NH}_{2}\right)$ of Lys166 from Cycl forms two $\mathrm{H}$-bonds with $\mathrm{CO}$ groups of Gly48 and Phe49 from RcY. The $\mathrm{NH}_{2}$ group of Gln163 from Cycl interacts with CO group of Phe 49 from RcY. CO group of Gln115 from Cycl interacts with $\mathrm{NH}_{2}$ group of Lys60 and $\mathrm{OH}$ of Ser53 from RcY. Main chain CO of Thr112 from Cyc1 interacts with main chain NH group of Gly147 from RcY. Main chain CO of Ala145 from RcY forms two H-bonds with main chain NH of Thr112 and main chain NH of Vall11 from Cycl (Figure 4(b) and Table S2). Our docking result identified a strong H-bond between His143 of RcY and Glu121 of Cyc1, which is in agreement with the X-ray crystallography study of Abergel et al. [16] (Figure 4(c)). Cycl contains two types of heme groups: heme $A$ and heme $B$. The former interacts with the $\mathrm{Cu}$ atom of $\mathrm{RcY}$, and the latter receives electron form heme $A$ and transfers it to the Cox. Analysis of the heme groups within the $\mathrm{Cycl}$ structure revealed that carboxyl groups of heme $A$ and heme $B$ interact directly with each other indicating a possible path of electron transfer to the next recipient protein (Figure $4(\mathrm{~d})$ ). The heme $A$ and heme $B$ exhibit high redox potentials of $+365 \mathrm{mV}$ and $+480 \mathrm{mV}$, respectively [60], which was found to be largely attributed to the environment of these two molecules. The CO groups of both the hemes were found to be involved in numerous $\mathrm{H}$-bond interactions with surrounding amino acids of $\mathrm{Cycl}$ (Table S3). Apart from H-bonds, the two hemes are stabilized by several nonbond interactions comprising residues Cys16, Cys19, His20, Pro33, Leu35, and Met64 for heme $A$ and Cys119, Cys122, His123, Pro134, Leu136, Leu147, and Met161 


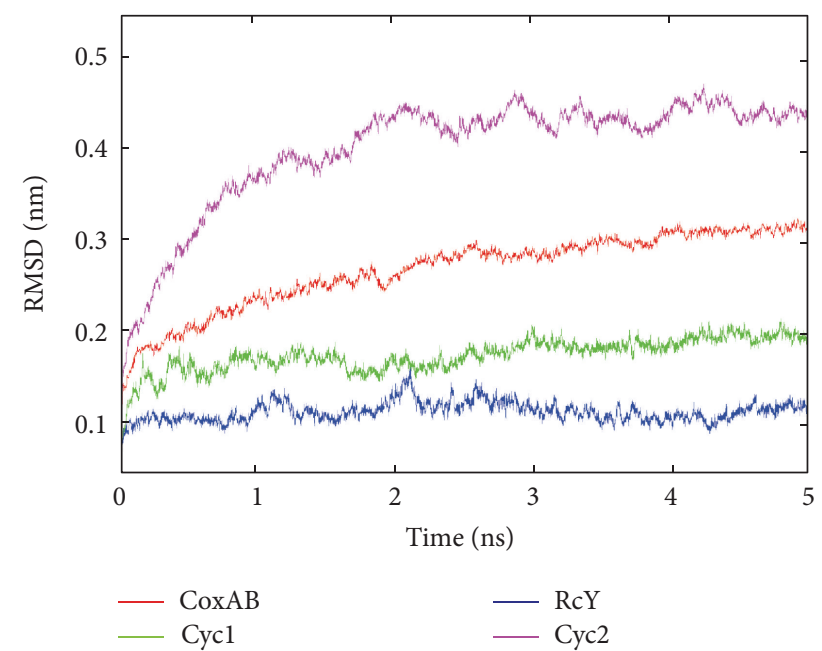

FIgure 3: Comparative RMSD graphs for Cyc2 (magenta), RcY (blue), Cycl (green), and CoxAB (red). The $x$-axis represents simulation time (in ps), and the $y$-axis indicates RMSD value (in $\mathrm{nm})$.

for heme B. A closer look on the number of H-bonds and the residues that participate in $\mathrm{H}$-bonds revealed that heme $A$ is stabilized by five $\mathrm{H}$-bonds and six direct nonbond interactions, whereas for heme $B$ seven $\mathrm{H}$-bonds and seven nonbond interaction were observed. Several past studies have suggested that RcY is present at the intermediate position between $\mathrm{Cyc2}$ and $\mathrm{Cycl}$ in A. ferrooxidans, as is shown in Figure 5. However, this model is probably specific to A. ferrooxidans, because of the fact that some acidophilic bacteria involved in $\mathrm{Fe}^{2+}$ oxidation reportedly do not possess the gene encoding $\mathrm{RcY}$ and that OM c type cytochrome is always located next to periplasmic c4 type cytochrome [61]. In such a situation, the OM cytochrome $\mathrm{c}$ and the periplasmic cytochrome c could be directly interacting in vivo while transferring electrons for energy metabolism [62].

3.5. Construction of CoxA and CoxB 3D Models. Cox is the terminal electron acceptor in the electron pathway of A. ferrooxidans. The diheme Cycl transfers electrons from OM Cyc2 to Cox's catalytic subunit for the reduction of $\mathrm{O}_{2}$ to form $\mathrm{H}_{2} \mathrm{O}[5,6]$. Cox is comprised of four subunits, namely, CoxA, CoxB, CoxC, and CoxD. CoxB is implicated in electron transfer from $\mathrm{Cycl}$ to CoxA subunit, where $\mathrm{O}_{2}$ is converted into a molecule of $\mathrm{H}_{2} \mathrm{O}$, whereas CoxC and CoxD mainly have a structural role $[5,36,63]$. Therefore, we constructed homology models for CoxA and CoxB subunits based on the respective subunits of Paracoccus denitrificans. BLAST search revealed that the target protein had $34 \%$ identity, $51 \%$ positives, and $73 \%$ query coverage with the template. The structure evaluation of the modeled protein using Ramachandran plot showed that $89.6 \%$ of residues fall within the most favored region and no residue was found in the disallowed region of the plot indicating conformational accuracy (Figure S6). The calculated Z-score of the modeled protein was -5.2 , which falls within the $Z$-score range of $\mathrm{X}$-ray determined structures of similar size (Figure S7).
Structure evaluation using Verify 3D showed that $80.09 \%$ of the residues had an average $3 \mathrm{D}-1 \mathrm{D}$ score $>0.2$. Normally, a score $>80 \%$ is a sign of satisfactory quality of the predicted model. The cofactors in the core region of the twelve TM domains, namely, one low spin heme a, one high spin heme a3, and one $\mathrm{Cu}$ atom, as reported in the experimental structure of the template, were obtained to the same coordinate space in the target model using structure alignment in DS 3.1. Similarly, homology model for CoxB was obtained from CoxB of Paracoccus denitrificans. The structure evaluation programs including Ramachandran plot indicated that $88.9 \%$ of residues were in most favored region with $0.9 \%$ residues in disallowed region. Verify 3D analysis revealed that $80.2 \%$ of the residues had averaged 3D-1D score $>0.2$, and ProSA-web calculation showed that the modeled structure had a $Z$-score that is within the range characteristic of native proteins of similar size. CoxB consists of a total of 254 amino acids with a calculated molecular weight of 28, 240 dalton [5]. The first 51 amino acids of this protein correspond to the standard signal sequence, and the mature protein is comprised of two $\mathrm{N}$ terminal TM domains and a $\mathrm{C}$ terminal periplasmic domain which are believed to transfer electrons to CoxA subunit $[36,63]$.

3.6. Interaction between $C y c 1$ and $\operatorname{Cox} A B$. Our study revealed that the $\mathrm{N}$ terminal TM helices of CoxB interact with 8th and 9th helices of CoxA subunit mainly through hydrophobic interactions involving side chains of both the subunits. The periplasmic domain of $\mathrm{CoxB}$ interacts with the periplasmic loops of CoxA through numerous $\mathrm{H}$-bonds. The $\mathrm{H}$-bond interaction between CoxB and CoxA subunits is shown in Table S4. As Cycl transfers electrons to the catalytic site of CoxA by interacting with the $\mathrm{Cu}$ center of $\mathrm{CoxB}$ [5], we carried out a detail analysis of the interaction between Cycl and CoxB. During MD simulation, the coordinates of CoxA and $\mathrm{CoxB}$ were grouped into a single index so that dynamic properties and energy profiles could be collectively obtained for comprehensive analysis. Stability of the CoxAB dimer was ascertained by $\mathrm{C} \alpha$ RMSD from the starting structure, which was calculated to be $0.31 \mathrm{~nm}$. Here, it may be noted that like $\mathrm{Cyc2}$, CoxAB is freed from the cytoplasmic end and hence required longer simulation time to reach equilibration. The overall compactness of the individual proteins with respect to their center of masses in the complex was analyzed by plotting radius of gyration $(\mathrm{Rg})$ values during $\mathrm{MD}$ simulation. Interestingly, we did not find an appreciable difference between the $\mathrm{Rg}$ values for $\mathrm{Cyc} 2$ and $\mathrm{Cox} \mathrm{AB}$ (Figure 6). Altogether, as exhibited in Figure 6, Cyc2 and CoxAB structures showed Rg values of $\sim 2.4 \mathrm{~nm}$. In contrast, both $\mathrm{RcY}$ and Cycl structures showed $\mathrm{Rg}$ values of $\sim 1.5 \mathrm{~nm}$. Since $\mathrm{Cyc} 2$ and $\mathrm{Cox} A B$ are comparative models, structural artifacts are inevitable to occur in the backbone or side chain conformations, and therefore, it might have resulted in higher Rg values during equilibration simulation. On the other hand, since RcY-Cyc1 complex is packed against both $\mathrm{Cyc} 2$ and $\mathrm{Cox} A B$ in the bacterial periplasm, it experienced greater compactness as indicated by lower Rg values. 


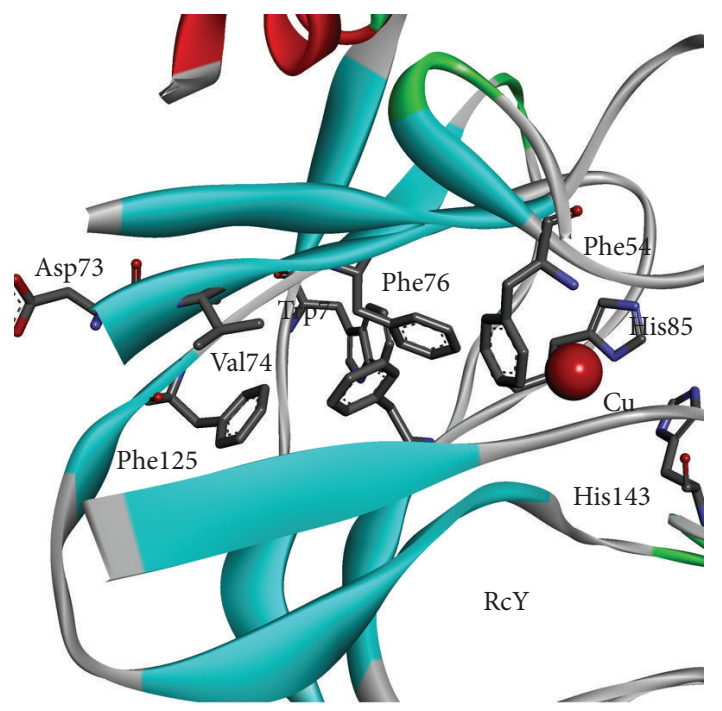

(a)

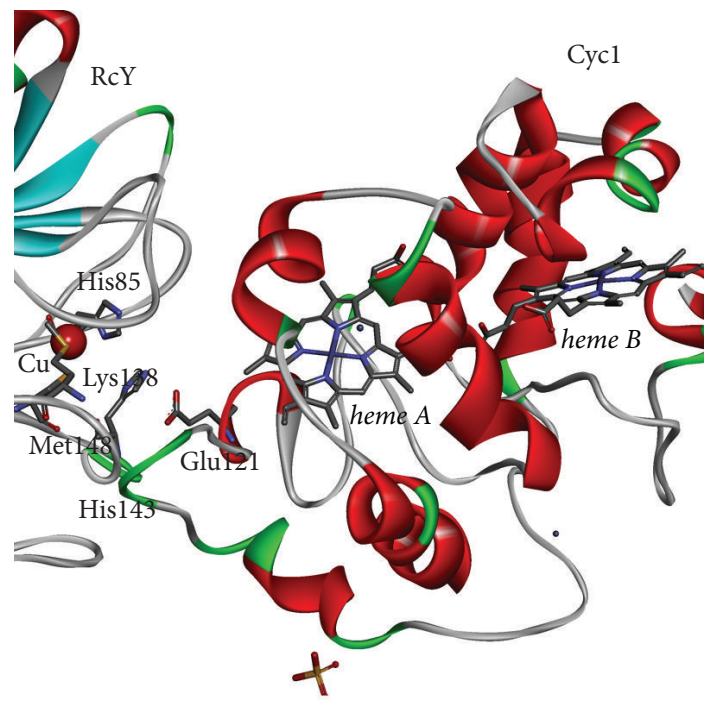

(c)

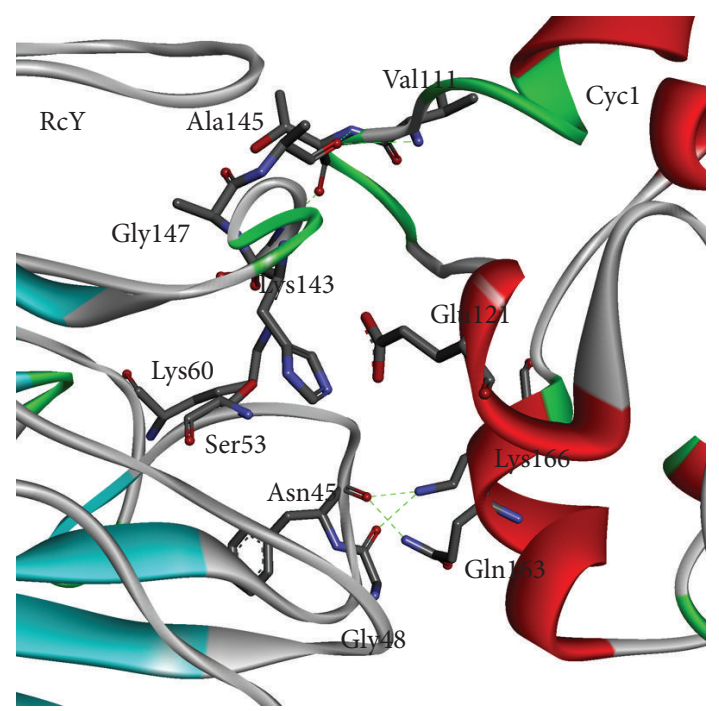

(b)

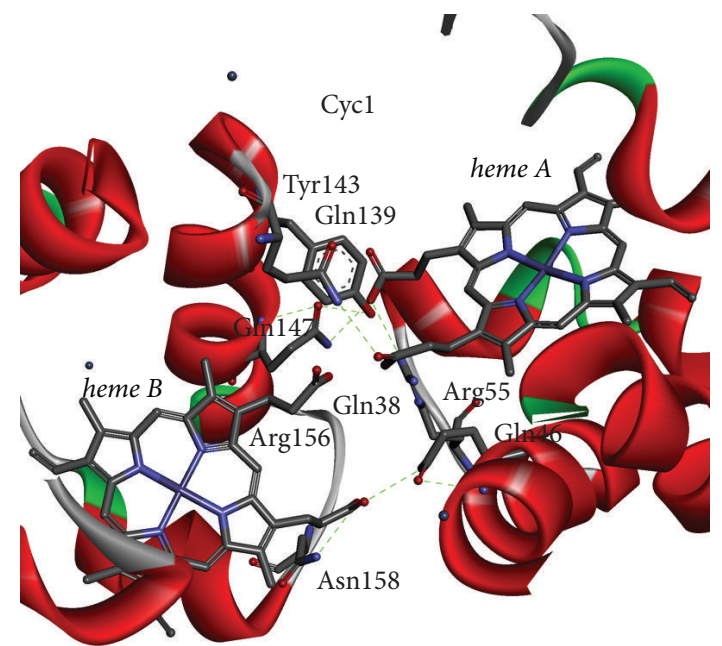

(d)

Figure 4: (a) The spatial distribution of core hydrophobic residues (stick) that facilitate electron transfer to RcY protein. (b) Illustration of $\mathrm{H}$-bonds (green dashed lines) formed between the residues of RcY-Cycl interacting complex. (c) The figure describes intermolecular contacts between the $\mathrm{Cu}$ center of $\mathrm{RcY}$ and the heme groups of $\mathrm{Cycl}$ through the His143-Glu121 interaction. (d) The figure depicts that the CO groups of heme $A$ and heme $B$ play crucial roles in their stability and electron transfer toward CoxB as revealed by strong intermolecular $\mathrm{H}$-bond interactions $(\sim 2.5 \AA)$.

Post-MD analysis for the average coordinates of CyclCoxAB revealed that $\mathrm{Cycl}$ forms a tight complex with the periplasmic domain of CoxB subunit involving numerous $\mathrm{H}$ bonds and electrostatic interactions. The H-bond network between these two proteins is as follows: the $\mathrm{C}$ terminal $\mathrm{CO}$ of Phe254 and $\mathrm{OH}$ of Thr157 from CoxB form $\mathrm{H}$-bonds with $\mathrm{NH}_{2}$ of Gln172 and epsilon nitrogen of His152 from Cycl, respectively. The main chain $\mathrm{NH}$ of Gly155 from CoxB interacts with the side chain $\mathrm{CO}$ of Asn153 from Cycl. One $\mathrm{Zn}^{2+}$ atom was found at the junction between CoxB and Cycl coordinated by His152 at a distance of $2.0 \AA$. The $\mathrm{Zn}^{2+}$ atom was found to be surrounded by Val159, Thr157, Gly155 of CoxB, and His152 of Cycl with a distance of 3.9, 2.1, 1.9, and $2.0 \AA$, respectively. The side chain $\mathrm{NH}_{2}$ of Lys146 from CoxB interacts with the main chain $\mathrm{CO}$ of Gly142 from $\mathrm{Cycl}$ through $\mathrm{H}$-bonds with a bond distance of $2.7 \AA$. The side chain $\mathrm{NH}$ of Gln58 from Cycl forms a comparatively loose $\mathrm{H}$-bond with the main chain $\mathrm{CO}$ of Asn228 from CoxB at a distance of 3.1 $\AA$ (Figure 7(a) and Table S5). Apart from these $\mathrm{H}$-bonds, the interaction between $\mathrm{Cycl}$ and $\mathrm{CoxB}$ structures was found to be stabilized by several hydrophobic residues comprising Val170, Tyr63, Tyr29, and Ile62 of Cycl and Val159, Trp145, and Val179 of CoxB.

A closer look into the binding interface of $\mathrm{Cycl}$ and $\mathrm{CoxB}$ revealed that CoxB possessed a stretch of seven residues, mostly hydrophobic in nature (145-WKWTFSY-151), which 


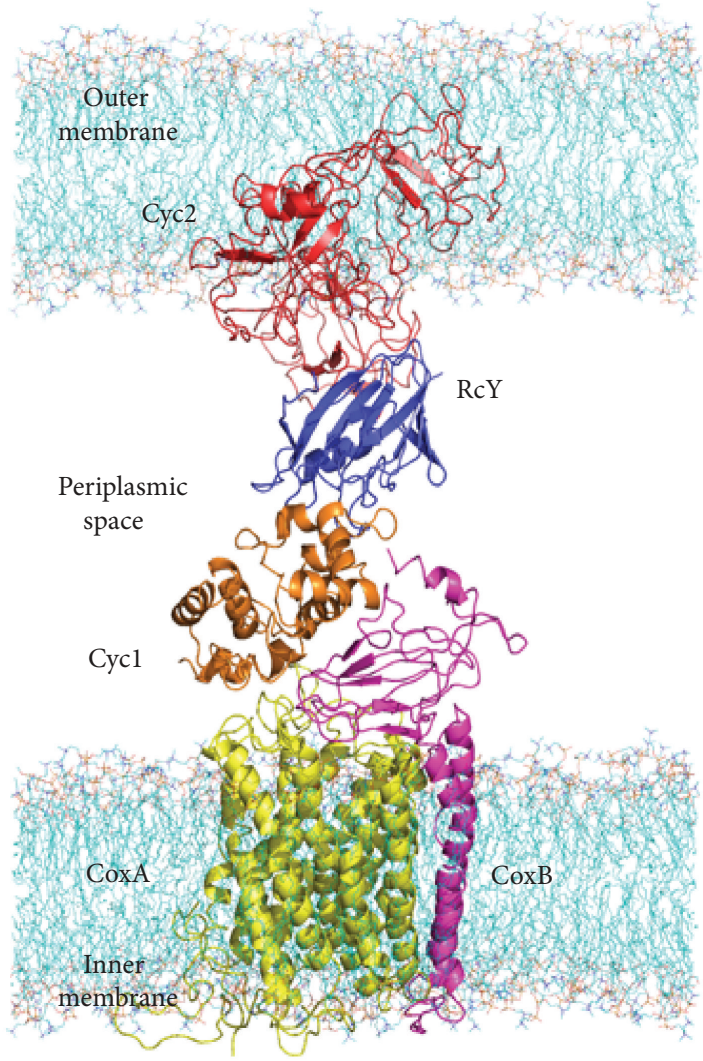

(a)

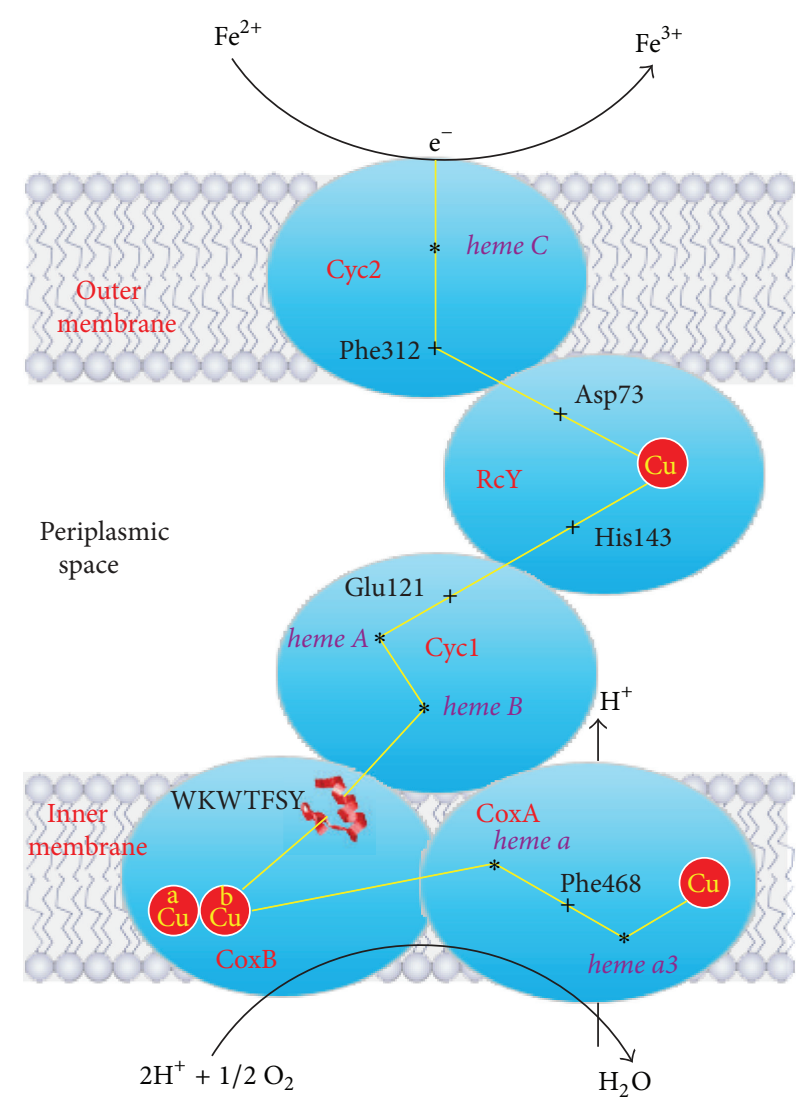

(b)

FIGURE 5: (a) The figure shows the membrane solvated model for docked respirasomes (Cyc2: red, RcY: blue, Cycl: golden, CoxB: purple, and CoxA: yellow). The figure was prepared using VMD software. (b) Graphical representation of electron-wire (green line) spanning Cyc2, RcY, $\mathrm{Cycl}$, and CoxAB subunits.

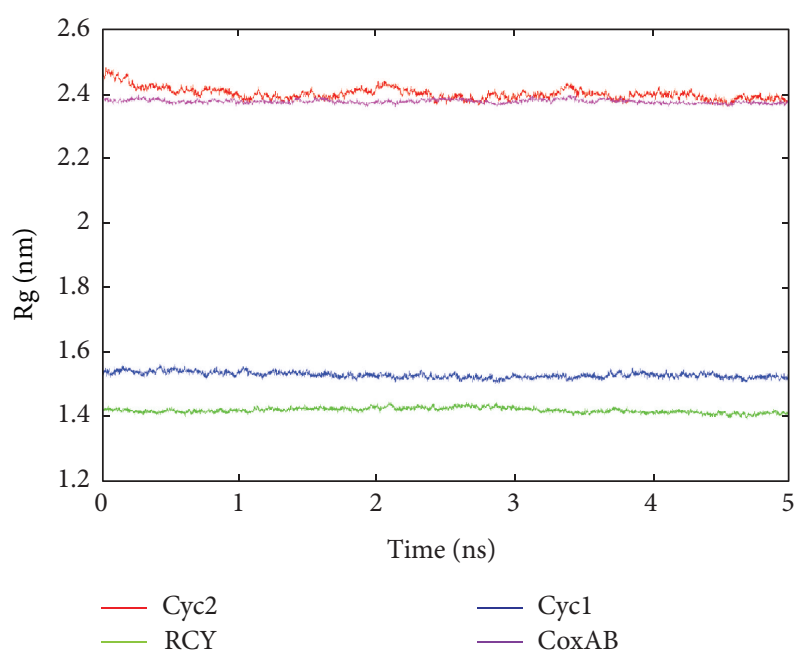

FIGURE 6: (a) Comparison of Rg values of each respirasome during MD simulation. The plot shows two groups of Rg values, one for Cyc2 and CoxAB, that is, $\sim 2.4 \AA$, and the other for RcY and Cycl, that is, $\sim 1.5 \AA$. The figure indicates that $\mathrm{RcY}$ and $\mathrm{Cycl}$ proteins were highly stable (low Rg value) in comparison to Cyc2 and CoxAB (high $\mathrm{Rg}$ value) during MD simulation. forms boundary line between surfaces of the two proteins. Lys146 and Thr148 of this motif were found to be responsible for stabilizing Cycl-CoxB complex by forming two strong H-bonds with Gly142 and Gly155 of Cycl, respectively (Figure 7(a)). These observations agree well the experimental data suggesting that the binuclear $\mathrm{Cu}$ center of $\mathrm{CoxB}$ subunit accepts electrons from heme $B$ of $\mathrm{Cycl}$ channeled through the WKWTFSY hydrophobic motif $[5,36,63]$. The CO group of heme $B$ might be the electron donor in this case. The two $\mathrm{Cu}$ atoms at CoxB periplasmic domain are separated from each other by a distance of $2.6 \AA$, and a disulfide bridge is formed between Cyc222 and Cyc226. CuA is coordinated by His181, Cyc226, and Met233, whereas CuB is coordinated by Cyc222 and His 230 (Figure 7(b)). From this $\mathrm{Cu}$ site, electron is passed to the CoxA catalytic site where $\mathrm{CO}$ of heme $a$, situated at a distance of $\sim 7 \AA$ from the $\mathrm{Cu}$ center of $\mathrm{CoxB}$, acts as the electron acceptor.

3.7. Stereochemistry of heme aa3 in the Catalytic Site of CoxA. CoxA of A. ferrooxidans contains heme $a$ at two different sites, each with a different function. The heme $a$ acts as an electron carrier and transfers electrons to heme a3, whereas the latter binds molecular $\mathrm{O}_{2}$ and reduces it into $\mathrm{H}_{2} \mathrm{O}$ with the help 


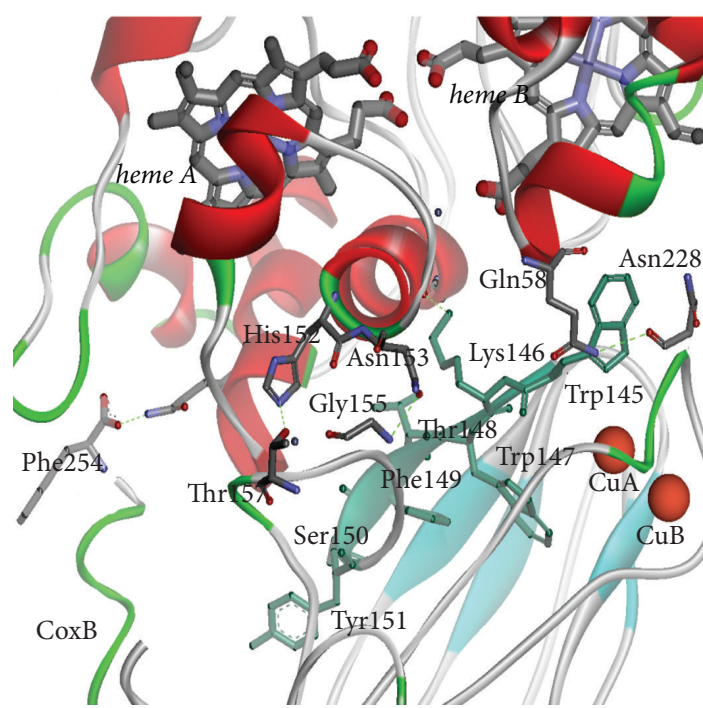

(a)

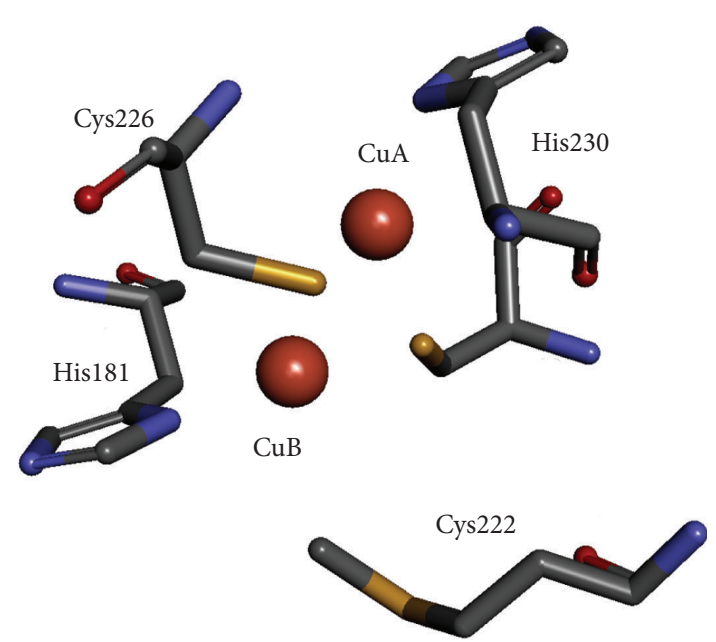

(b)

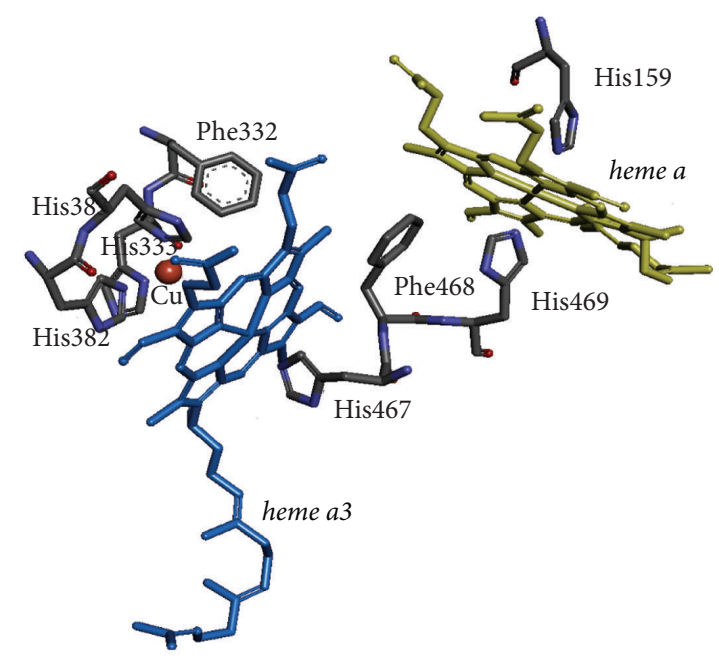

(c)

Figure 7: (a) The figure represents the interaction between Cycl and CoxB. The hydrophobic motif 145-WKWTFSY-151 essential for electron transfer is highlighted in green color. H-bonds are shown as dashed lines, heme groups of Cycl are represented as stick models, and CuA and $\mathrm{CuB}$ are shown as space fill models in red color. (b) The schematic representation of binuclear $\mathrm{Cu}$ center of CoxB showing the $\mathrm{CuA}$ coordinated by Cys222, Cys226, and His 230 and CuB coordinated by Met223, His181, Cys222, and Cys226. The Cu site of CoxB plays an important role in further electron transfer to the catalytic site of CoxA. (c) The figure illustrates the interaction between heme $a$ and heme a3 within the catalytic site of CoxA.

of an electron received from extracellular environment (Figure 7(c)). While Fe of heme $a$ is hexacoordinated, four from the porphyrin ring of heme $a$ and the remaining two from His159 and His469 of CoxA, Fe of heme a3 is coordinated by five different nitrogen atoms, four from the nitrogen atoms of the porphyrin ring and one from His 467 of CoxA, thus leaving the sixth site available to bind $\mathrm{O}_{2}$ [64]. The heme a3, in turn, is involved in reduction of $\mathrm{O}_{2}$ to form $\mathrm{H}_{2} \mathrm{O}$ with the help of a $\mathrm{Cu}$ cofactor. The $\mathrm{Cu}$ atom is coordinated by His333, His382, and His383 (Figure 7(b)). One of the $\mathrm{Cu}$ coordinating histidine amino acids might play electrophilic role required for the catalysis. The details of stabilizing $\mathrm{H}$-bonds between heme groups and amino acids of CoxA are presented in Table S6. In addition to this, it was proposed that the conserved Phe 468 found in between His467 and His469 of CoxA might be important for the transfer of electrons from heme a to heme $a 3[65,66]$, and our simulation results support this hypothesis. During the $5 \mathrm{~ns}$ MD simulation, two heme groups were found to be quite stable, probably due to the hydrophobic effect put forth by Phe 468 as indicated by the RMSD graph (Figure 8 ). The results indicated that heme a experienced less fluctuation during MD simulation because it is connected to His159 and His469 from both sides, whereas heme a3 experienced comparatively larger fluctuation due to the absence of $\mathrm{O}_{2}$ at one end. The stability of heme aa3 binding region was 


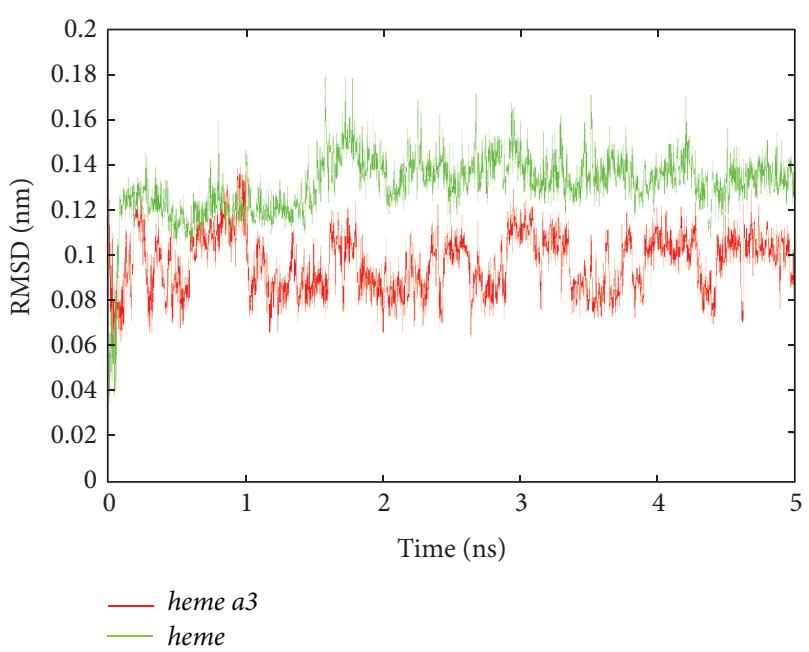

FIGURE 8: Comparative RMSD graphs for heme a (green) and heme $a 3$ (red). The $x$ axis represents simulation time (in ps) and the $y$ axis indicates RMSD value (in $\mathrm{nm}$ ). Although the RMSD value of heme $a(0.15 \mathrm{~nm})$ was found to be greater than that of heme a3 $(0.9 \mathrm{~nm})$, it maintains stability throughout the simulation.

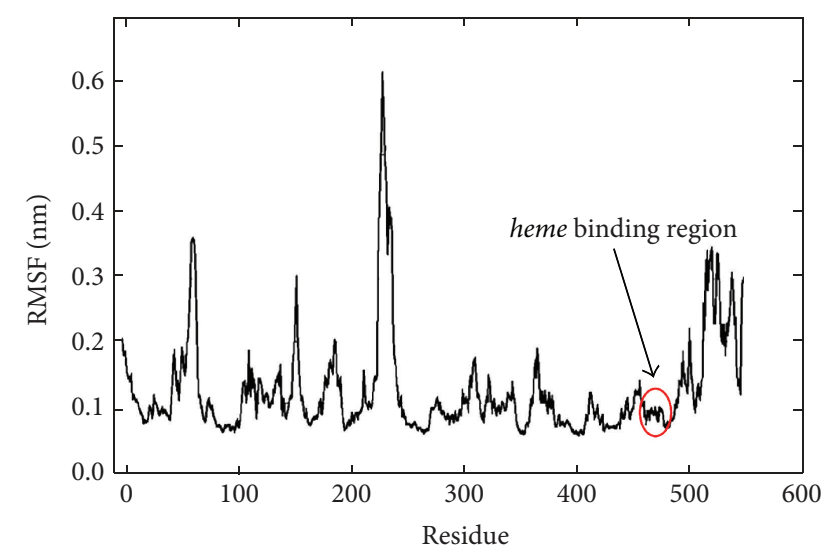

FIgURE 9: The figure shows fluctuation of CoxA residues during MD simulation. The fluctuation of heme binding region (residues 461-471), specifically, the residues His467, Phe468, and His469, was found to be very low indicating their structural stability.

ascertained by observing root mean square fluctuation of backbone residues during MD simulation (Figure 9). The residues that connect heme a to heme a3, that is, His467, Phe468, and His469, were found to be completely stable during simulation. Our modeling and simulation results suggest that the residues of Cox involved in stabilizing heme $a a 3$ are found at the upper side of cytoplasmic membrane near the periplasm. From this observation, we can postulate that $\mathrm{O}_{2}$ reduction takes place at a $\mathrm{pH}$ nearly equivalent to periplasmic $\mathrm{pH}$ of $3.0[67,68]$.

\section{Conclusions}

The interdependence among the docked structures was first observed when $\mathrm{OM}$ and Phe312. At the interacting surface,
Asp73 of RcY was considered to be involved in direct electron transfer between two complexes, which might be facilitated by physical interactions of Phe312 and Asp73. A detailed analysis revealed that the hydrophobic residues enclosed within core region of $\mathrm{RcY}$ form a passage for electrons to reach the $\mathrm{Cu}$ center. Subsequent docking of Cycl with Cyc2-RcY complex indicated that His143 of RcY associates with Glu121 of Cycl, suggesting a probable channel of electron transfer between the two proteins. The heme $A$ and heme $B$ of Cycl play crucial roles in transferring electron received from $\mathrm{RcY}$ to the binuclear $\mathrm{Cu}$ center of $\mathrm{CoxB}$ through the aromatic/hydrophobic sequence motif, that is, 145-WKWTFSY-151. Phe468 was found to stabilize heme a and heme $a 3$ byhydrophobic interaction. Our results provide a comprehensive insight on the structural organization of the respiratory supercomplex and on the probable path of electrons within downhill pathway of A. ferrooxidans. Altogether, this computational model can be used to understand the popular electron wire concept of metalloproteins.

\section{Conflict of Interests}

The authors declare that no competing interests exist with any commercial bodies.

\section{Acknowledgments}

The authors are thankful to Department of Biotechnology, Government of India, for providing computational facility to carry out the present research. The authors also declare that valid license has been acquired for the commercial software used in this study.

\section{References}

[1] L. R. Croal, J. A. Gralnick, D. Malasarn, and D. K. Newman, "The genetics of geochemistry," Annual Review of Genetics, vol. 38, pp. 175-202, 2004.

[2] T. Rohwerder, T. Gehrke, K. Kinzler, and W. Sand, "Bioleaching review part A: progress in bioleaching: fundamentals and mechanisms of bacterial metal sulfide oxidation," Applied Microbiology and Biotechnology, vol. 63, no. 3, pp. 239-248, 2003.

[3] A. Elbehti, G. Brasseur, and D. Lemesle-Meunier, "First evidence for existence of an uphill electron transfer through the $b c_{1}$ and NADH-Q oxidoreductase complexes of the acidophilic obligate chemolithotrophic ferrous ion-oxidizing bacterium Thiobacillus ferrooxidans," Journal of Bacteriology, vol. 182, no. 12, pp. 3602-3606, 2000.

[4] G. Brasseur, G. Levican, V. Bonnefoy, D. Holmes, E. Jedlicki, and D. Lemesle-Meunier, "Apparent redundancy of electron transfer pathways via $b c_{1}$ complexes and terminal oxidases in the extremophilic chemolithoautotrophic Acidithiobacillus ferrooxidans," Biochimica et Biophysica Acta, vol. 1656, no. 2-3, pp. 114-126, 2004.

[5] C. Appia-Ayme, N. Guiliani, J. Ratouchniak, and V. Bonnefoy, "Characterization of an operon encoding two $c$-type cytochromes, an aa3-type cytochrome oxidase, and rusticyanin in Thiobacillus ferrooxidans ATCC 33020," Applied and Environmental Microbiology, vol. 65, no. 11, pp. 4781-4787, 1999. 
[6] A. Yarzábal, G. Brasseur, J. Ratouchniak et al., “The highmolecular-weight cytochrome $c$ Cyc2 of Acidithiobacillus ferrooxidans is an outer membrane protein," Journal of Bacteriology, vol. 184, no. 1, pp. 313-317, 2002.

[7] G. Malarte, G. Leroy, E. Lojou, C. Abergel, M. Bruschi, and M. T. Giudici-Orticoni, "Insight into molecular stability and physiological properties of the diheme cytochrome $\mathrm{CYC}_{41}$ from the acidophilic bacterium Acidithiobacillus ferrooxidans," Biochemistry, vol. 44, no. 17, pp. 6471-6481, 2005.

[8] A. Bengrine, N. Guiliani, C. Appia-Ayme et al., "Sequence and expression of the rusticyanin structural gene from Thiobacillus ferrooxidans ATCC33020 strain," Biochimica et Biophysica Acta, vol. 1443, no. 1-2, pp. 99-112, 1998.

[9] P. Bruscella, C. Appia-Ayme, G. Levicán et al., "Differential expression of two $b c_{1}$ complexes in the strict acidophilic chemolithoautotrophic bacterium Acidithiobacillus ferrooxidans suggests a model for their respective roles in iron or sulfur oxidation," Microbiology, vol. 153, no. 1, pp. 102-110, 2007.

[10] W. J. Ingledew, "Thiobacillus ferrooxidans. The bioenergetics of an acidophilic chemolithotroph," Biochimica et Biophysica Acta, vol. 683 , no. 2 , pp. $89-117,1982$.

[11] D. Holmes and V. Bonnefoy, "Genetic and bioinformatic insights into iron and sulfur oxidation mechanisms of bioleaching organisms," in Biomining, D. E. Rawlings, E. Douglas, and D. B. Johnson, Eds., pp. 281-307, Springer, Berlin, Germany, 2007.

[12] A. Yarzábal, C. Appia-Ayme, J. Ratouchniak, and V. Bonnefoy, "Regulation of the expression of the Acidithiobacillus ferrooxidans rus operon encoding two cytochromes $c$, a cytochrome oxidase and rusticyanin," Microbiology, vol. 150, no. 7, pp. 21132123, 2004.

[13] C. Castelle, M. Guiral, G. Malarte et al., "A new ironoxidizing $/ \mathrm{O}_{2}$-reducing supercomplex spanning both inner and outer membranes, isolated from the extreme acidophile Acidithiobacillus ferrooxidans," Journal of Biological Chemistry, vol. 283, no. 38, pp. 25803-25811, 2008.

[14] M. L. Barrett, I. Harvey, M. Sundararajan et al., "Atomic resolution crystal structures, EXAFS, and quantum chemical studies of rusticyanin and its two mutants provide insight into its unusual properties," Biochemistry, vol. 45, no. 9, pp. 29272939, 2006.

[15] R. L. Walter, S. E. Ealick, A. M. Friedman, R. C. Blake II, P. Proctor, and M. Shoham, "Multiple wavelength anomalous diffraction (MAD) crystal structure of rusticyanin: a highly oxidizing cupredoxin with extreme acid stability," Journal of Molecular Biology, vol. 263, no. 5, pp. 730-751, 1996.

[16] C. Abergel, W. Nitschke, G. Malarte, M. Bruschi, J.-M. Claverie, and M.-T. Giudici-Orticoni, "The structure of Acidithiobacillus ferrooxidans $c_{4}$-cytochrome: a model for complex-induced electron transfer tuning," Structure, vol. 11, no. 5, pp. 547-555, 2003.

[17] B. P. Mukhopadhyay, B. Ghosh, H. R. Bairagya, T. K. Nandi, B. Chakrabarti, and A. K. Bera, "Molecular modeling of the ternary complex of rusticyanin-cytochrome $c_{4}$-cytochrome oxidase: an insight to possible $\mathrm{H}$-bond mediated recognition and electron transfer reaction in T. ferrooxidans," Journal of Biomolecular Structure and Dynamics, vol. 25, no. 5, pp. 543$551,2008$.

[18] B. P. Mukhopadhyay, B. Ghosh, H. R. Bairagya, A. K. Bera, T. K. Nandi, and S. B. Das, "Modeling study of rusticyanincytochrome $c_{4}$ complex: an insight to possible H-bond mediated recognition and electron-transfer process," Journal of Biomolecular Structure and Dynamics, vol. 25, no. 2, pp. 157-164, 2007.
[19] B. P. Mukhopadhyay, B. Ghosh, H. R. Bairagya, A. K. Bera, and R. K. Roy, "Conserved water molecular dynamics of the different X-ray structures of rusticyanin: an unique aquation potentiality of the ligand bonded $\mathrm{Cu}^{++}$center," Journal of Biomolecular Structure and Dynamics, vol. 24, no. 4, pp. 369377, 2007.

[20] S. F. Altschul, W. Gish, W. Miller, E. W. Myers, and D. J. Lipman, "Basic local alignment search tool," Journal of Molecular Biology, vol. 215, no. 3, pp. 403-410, 1990.

[21] J. U. Bowie, R. Luthy, and D. Eisenberg, "A method to identify protein sequences that fold into a known three-dimensional structure," Science, vol. 253, no. 5016, pp. 164-170, 1991.

[22] L. A. Kelley and M. J. E. Sternberg, "Protein structure prediction on the Web: a case study using the Phyre server," Nature Protocols, vol. 4, no. 3, pp. 363-371, 2009.

[23] J. Shi, T. L. Blundell, and K. Mizuguchi, "FUGUE: sequencestructure homology recognition using environment-specific substitution tables and structure-dependent gap penalties," Journal of Molecular Biology, vol. 310, no. 1, pp. 243-257, 2001.

[24] K. Karplus, R. Karchin, J. Draper et al., "Combining localstructure, fold-recognition, and new fold methods for protein structure prediction," Proteins, vol. 53, supplement 6, pp. 491496, 2003.

[25] A. Lobley, M. I. Sadowski, and D. T. Jones, "pGenTHREADER and pDomTHREADER: new methods for improved protein fold recognition and superfamily discrimination," Bioinformatics, vol. 25, no. 14, pp. 1761-1767, 2009.

[26] M. A. Kurowski and J. M. Bujnicki, "GeneSilico protein structure prediction meta-server," Nucleic Acids Research, vol. 31, no. 13, pp. 3305-3307, 2003.

[27] J. Lundström, L. Rychlewski, J. Bujnicki, and A. Elofsson, "Pcons: a neural-network-based consensus predictor that improves fold recognition," Protein Science, vol. 10, no. 11, pp. 2354-2362, 2001.

[28] D. T. Jones, "Protein secondary structure prediction based on position-specific scoring matrices," Journal of Molecular Biology, vol. 292, no. 2, pp. 195-202, 1999.

[29] N. Treiber, D. J. Reinert, I. Carpusca, K. Aktories, and G. E. Schulz, "Structure and mode of action of a mosquitocidal holotoxin," Journal of Molecular Biology, vol. 381, no. 1, pp. 150159, 2008.

[30] D. van der Spoel, E. Lindahl, B. Hess, G. Groenhof, A. E. Mark, and H. J. C. Berendsen, "GROMACS: fast, flexible, and free," Journal of Computational Chemistry, vol. 26, no. 16, pp. 17011718, 2005.

[31] M. Wiederstein and M. J. Sippl, "ProSA-web: interactive web service for the recognition of errors in three-dimensional structures of proteins," Nucleic Acids Research, vol. 35, supplement 2, pp. W407-W410, 2007.

[32] T. E. Meyer, "Evolution and classification of c-type cytochromes," in Cytochrome C: A Multidisciplinary Approach, R. A. Scott and A. G. Mauk, Eds., University Science Books, Sausalito, Calif, USA, 1995.

[33] E. E. Bolton, Y. Wang, P. A. Thiessen, and S. H. Bryant, "PubChem: integrated platform of small molecules and biological activities," Annual Reports in Computational Chemistry, vol. 4, pp. 217-241, 2008.

[34] D. Schneidman-Duhovny, Y. Inbar, R. Nussinov, and H. J. Wolfson, "PatchDock and SymmDock: servers for rigid and symmetric docking," Nucleic Acids Research, vol. 33, supplement 2, pp. W363-W367, 2005. 
[35] D. Duhovny, R. Nussinov, and H. J. Wolfson, "Efficient unbound docking of rigid molecules," in Algorithms in Bioinformatics: Second International Workshop, WABI 2002, Rome, Italy, September 17-21, 2002, Proceedings, R. Guigo and D. Gusfeld, Eds., vol. 2452 of Lecture Notes in Computer Science, pp. 185200, Springer, 2002.

[36] S. Iwata, C. Ostermeier, B. Ludwig, and H. Michel, "Structure at $2.8 \AA$ resolution of cytochrome $c$ oxidase from Paracoccus denitrificans," Nature, vol. 376, pp. 660-669, 2002.

[37] C. Neale, W. F. D. Bennett, D. P. Tieleman, and R. Pomès, "Statistical convergence of equilibrium properties in simulations of molecular solutes embedded in lipid bilayers," Journal of Chemical Theory and Computation, vol. 7, no. 12, pp. 4175-4188, 2011.

[38] C. Kandt, W. L. Ash, and D. P. Tieleman, "Setting up and running molecular dynamics simulations of membrane proteins," Methods, vol. 41, no. 4, pp. 475-488, 2007.

[39] J. F. Nagle, "Area/lipid of bilayers from NMR," Biophysical Journal, vol. 64, no. 5, pp. 1476-1481, 1993.

[40] O. Berger, O. Edholm, and F. Jähnig, "Molecular dynamics simulations of a fluid bilayer of dipalmitoylphosphatidylcholine at full hydration, constant pressure, and constant temperature," Biophysical Journal, vol. 72, no. 5, pp. 2002-2013, 1997.

[41] W. Humphrey, A. Dalke, and K. Schulten, "VMD: visual molecular dynamics," Journal of Molecular Graphics, vol. 14, no. 1, pp. 33-38, 1996.

[42] D. Baker and A. Sali, "Protein structure prediction and structural genomics," Science, vol. 294, no. 5540, pp. 93-96, 2001.

[43] M. J. L. Schoonman, R. M. A. Knegtel, and P. D. J. Grootenhuis, "Practical evaluation of comparative modelling and threading methods," Computers and Chemistry, vol. 22, no. 5, pp. 369-375, 1998.

[44] K. L. Tkaczuk, “Trm13p, the tRNA:Xm4 modification enzyme from Saccharomyces cerevisiae is a member of the Rossmannfold MTase superfamily: prediction of structure and active site," Journal of Molecular Modeling, vol. 16, no. 3, pp. 599-606, 2010.

[45] K. A. Majorek and J. M. Bujnicki, "Modeling of Escherichia coli Endonuclease V structure in complex with DNA," Journal of Molecular Modeling, vol. 15, no. 2, pp. 173-182, 2009.

[46] A. N. Ramli, N. M. Mahadi, M. S. Shamsir et al., "Structural prediction of a novel chitinase from the psychrophilic Glaciozyma antarctica PI12 and an analysis of its structural properties and function," Journal of Computer Aided Molecular Design, vol. 26, no. 8, pp. 947-996, 2012.

[47] P. Anand, S. Sankaran, S. Mukherjee et al., "Structural annotation of Mycobacterium tuberculosis proteome," PLoS ONE, vol. 6, no. 10, Article ID e27044, 2011.

[48] M. Ferrer, A. Ghazi, A. Beloqui et al., "Functional metagenomics unveils a multifunctional glycosyl hydrolase from the family 43 catalysing the breakdown of plant polymers in the calf rumen," PLoS ONE, vol. 7, no. 6, Article ID e38134, 2012.

[49] F. Rückert, G. Dawelbait, C. Winter et al., "Examination of apoptosis signaling in pancreatic cancer by computational signal transduction analysis," PLOS ONE, vol. 5, no. 8, Article ID e12243, 2010.

[50] G. N. Ramachandran, C. Ramakrishnan, and V. Sasisckharan, "Stereochemistry of polypeptide chain configurations," Journal of Molecular Biology, vol. 7, pp. 95-99, 1963.

[51] F. van Ommen Kloeke, R. D. Bryant, and E. J. Laishley, "Localization of cytochromes in the outer membrane of Desulfovibrio vulgaris (hildenborough) and their role in anaerobic biocorrosion," Anaerobe, vol. 1, no. 6, pp. 351-358, 1995.
[52] S. Gaspard, F. Vazquez, and C. Holliger, "Localization and solubilization of the iron(III) reductase of Geobacter sulfurreducens," Applied and Environmental Microbiology, vol. 64, no. 9, pp. 3188-3194, 1998.

[53] C. R. Myers and J. M. Myers, "Localization of cytochromes to the outer membrane of anaerobically grown Shewanella putrefaciens MR-1," Journal of Bacteriology, vol. 174, no. 11, pp. 3429-3438, 1992.

[54] J. M. Myers and C. R. Myers, "Role for outer membrane cytochromes OmcA and OmcB of Shewanella putrefaciens MR-1 in reduction of manganese dioxide," Applied and Environmental Microbiology, vol. 67, no. 1, pp. 260-269, 2001.

[55] W. J. Ingledew, J. C. Cox, and P. J. Halling, "A proposed mechanism for energy conservation during $\mathrm{Fe}^{2+}$ oxidation by Thiobacillus ferro-oxidans: chemiosmotic coupling to net $\mathrm{H}^{+}$ influx," FEMS Microbiology Letters, vol. 2, no. 4, pp. 193-197, 1977.

[56] M. Ronk, J. E. Shively, E. A. Shute, and R. C. Blake II, "Amino acid sequence of the blue copper protein rusticyanin from Thiobacillus ferrooxidans," Biochemistry, vol. 30, no. 39, pp. 9435-9442, 1991.

[57] D. R. Casimiro, A. Toy-Palmer, R. C. Blake II, and H. J. Dyson, "Gene synthesis, high-level expression, and mutagenesis of Thiobacillus ferrooxidans rusticyanin: His 85 is a ligand to the blue copper center," Biochemistry, vol. 34, no. 20, pp. 66406648, 1995.

[58] N. Liang, G. J. Pielak, A. G. Mauk, M. Smith, and B. M. Hoffman, "Yeast cytochrome $c$ with phenylalanine or tyrosine at position 87 transfers electrons to (zinc cytochrome $c$ peroxidase) ${ }^{+}$at a rate ten thousand times that of the serine- 87 or glycine- 87 variants," Proceedings of the National Academy of Sciences of the United States of America, vol. 84, no. 5, pp. 1249-1252, 1987.

[59] C. S. Miles, N. Rouviere-Fourmy, F. Lederer et al., "Tyr-143 facilitates interdomain electron transfer in flavocytochrome $b_{2}$," Biochemical Journal, vol. 285, part 1, pp. 187-192, 1992.

[60] M.-T. Giudici-Orticoni, G. Leroy, W. Nitschke, and M. Bruschi, "Characterization of a new dihemic $c_{4}$-type cytochrome isolated from Thiobacillus ferrooxidans," Biochemistry, vol. 39, no. 24, pp. 7205-7211, 2000.

[61] C. Jeans, S. W. Singer, C. S. Chan et al., "Cytochrome 572 is a conspicuous membrane protein with iron oxidation activity purified directly from a natural acidophilic microbial community," The ISME Journal, vol. 2, no. 5, pp. 542-550, 2008.

[62] W. J. Ingledew and J. G. Cobley, "A potentiometric and kinetic study on the respiratory chain of ferrous-iron-grown Thiobacillus ferrooxidans," Biochimica et Biophysica Acta, vol. 590, no. 2, pp. 141-158, 1980.

[63] P. Lappalainen, N. J. Watmough, C. Greenwood, and M. Saraste, "Electron transfer between cytochrome $c$ and the isolated $\mathrm{CuA}$ domain: identification of substrate-binding residues in cytochrome $c$ oxidase," Biochemistry, vol. 34, no. 17, pp. 58245830, 1995.

[64] S. Yoshikawa, K. Shinzawa-Itoh, R. Nakashima et al., "Redoxcoupled crystal structural changes in bovine heart cytochrome c oxidase," Science, vol. 280, no. 5370, pp. 1723-1729, 1998.

[65] C. Ostermeier, S. Iwata, and H. Michel, "Cytochrome c oxidase," Current Opinion in Structural Biology, vol. 6, no. 4, pp. 460-466, 1996.

[66] M. Saraste, J. Castresana, D. Higgins, M. Lbben, and M. Wilmanns, "Evolution of cytochrome oxidase," in Origin and Evolution of Biological Energy Conversion, H. Baltscheffsky, Ed., pp. 255-289, Wiley-VCH, New York, NY, USA, 1994. 
[67] M. Kai, T. Yano, Y. Fukumori, and T. Yamanaka, "Cytochrome oxidase of an acidophilic iron-oxidizing bacterium, Thiobacillus ferrooxidans, functions at $\mathrm{pH}$ 3.5," Biochemical and Biophysical Research Communications, vol. 160, no. 2, pp. 839-843, 1989.

[68] M. Kai, T. Yano, H. Tamegai, Y. Fukumori, and T. Yamanaka, "Thiobacillus ferrooxidans cytochrome $c$ oxidase: purification, and molecular and enzymatic features," Journal of Biochemistry, vol. 112, no. 6, pp. 816-821, 1992. 

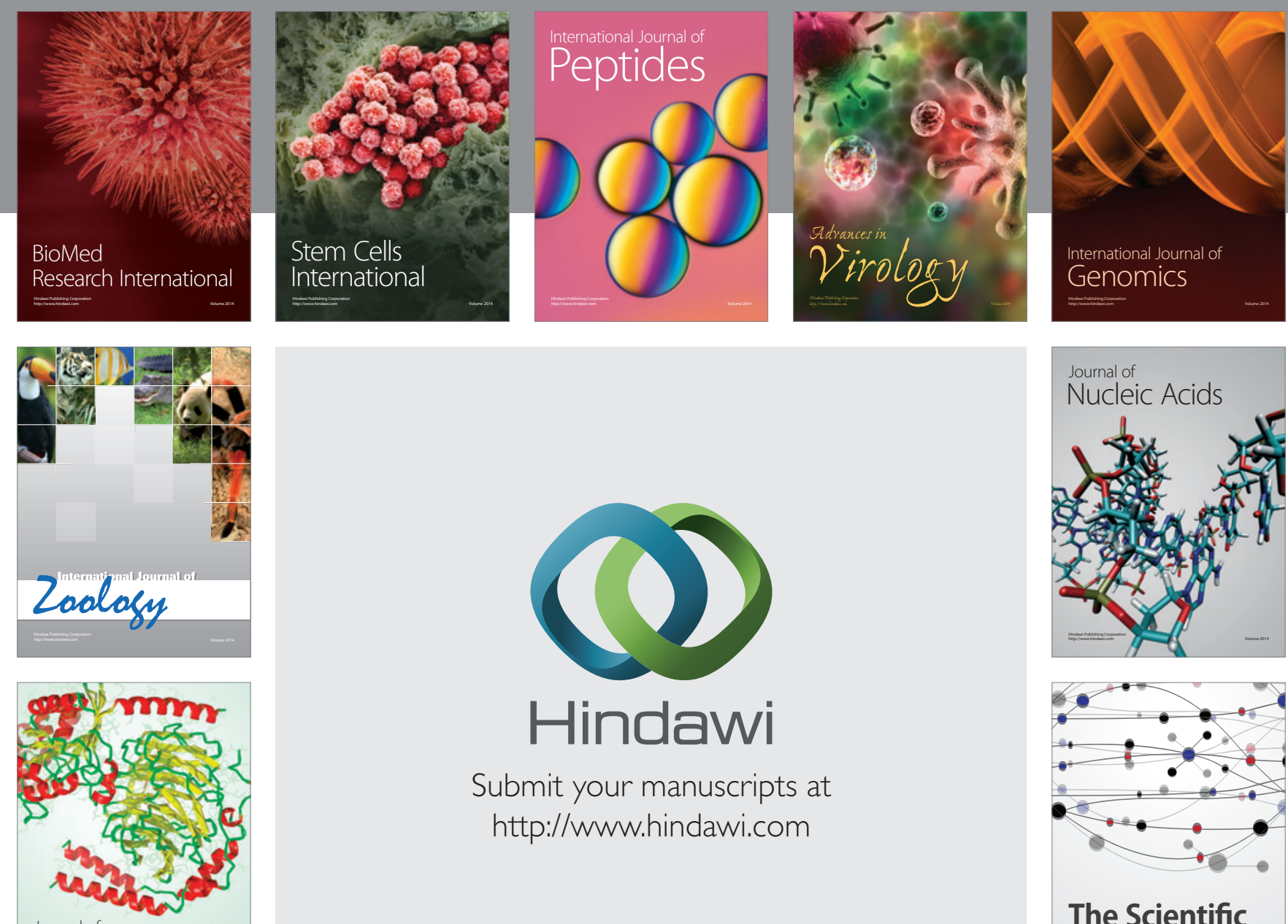

Submit your manuscripts at

http://www.hindawi.com

Journal of
Signal Transduction
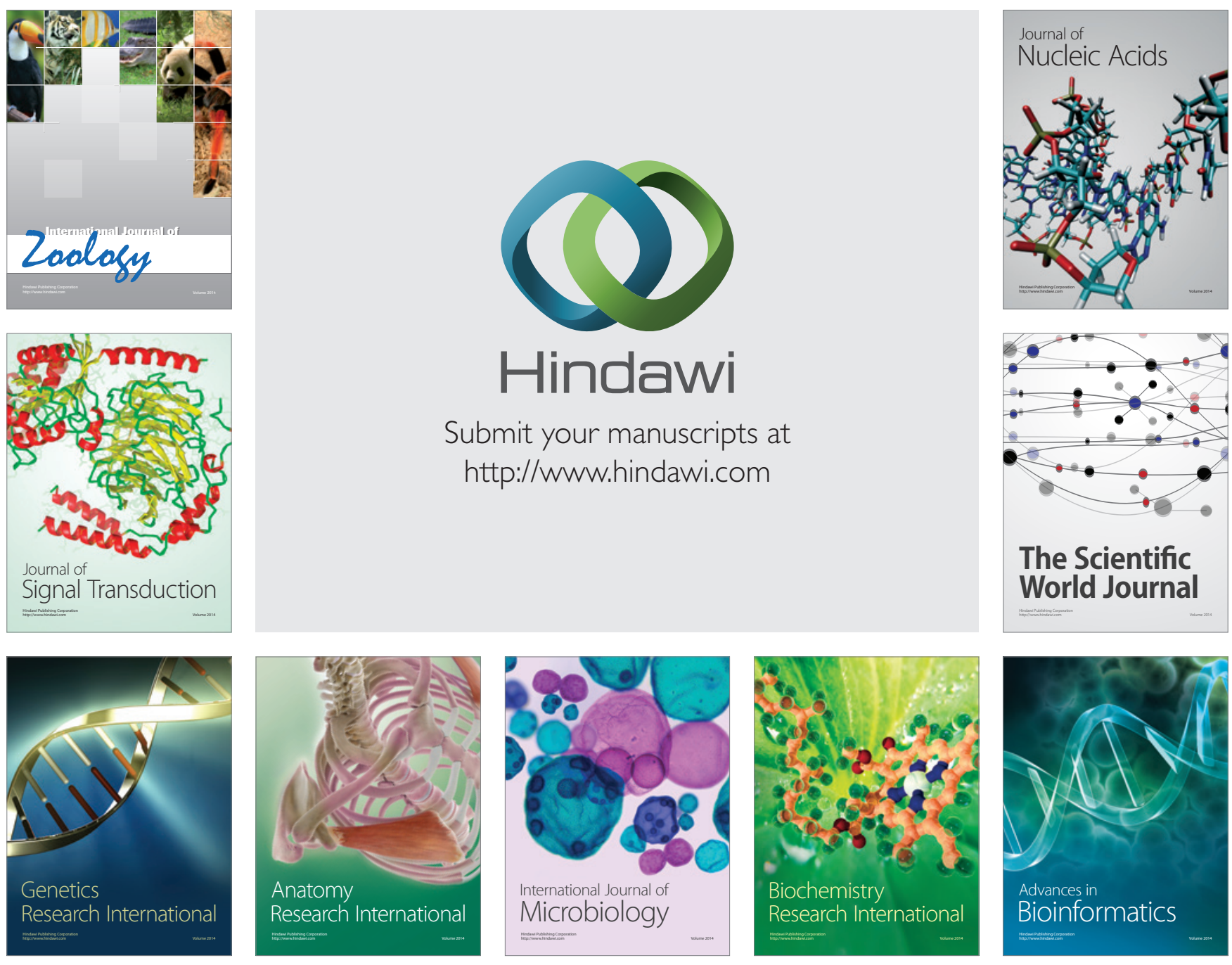

The Scientific World Journal
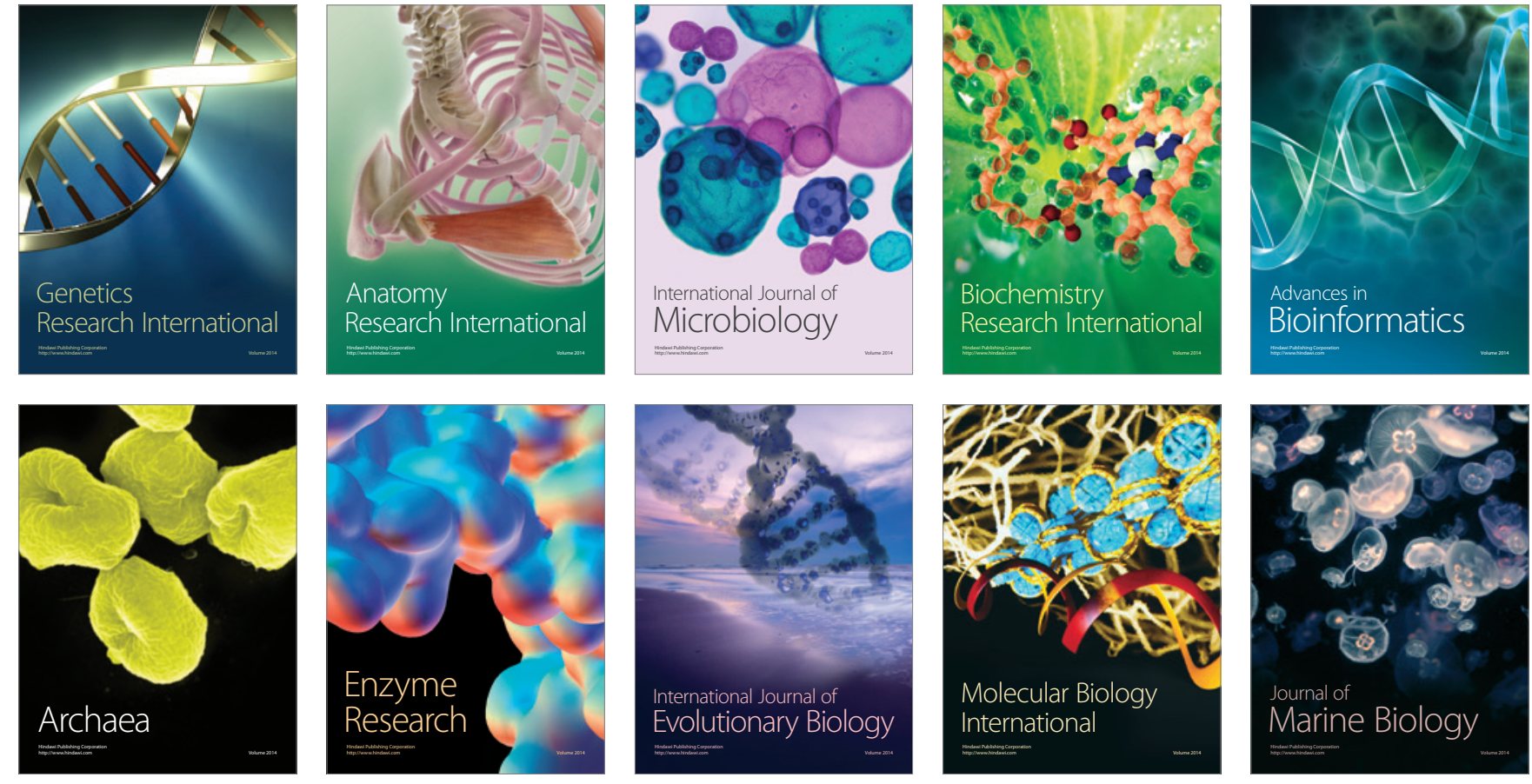